\title{
Human iPS cell-derived alveolar epithelium repopulates lung extracellular matrix
}

\author{
Mahboobe Ghaedi, ${ }^{1}$ Elizabeth A. Calle, ${ }^{1}$ Julio J. Mendez, ${ }^{1}$ Ashley L. Gard, ${ }^{1}$ Jenna Balestrini, ${ }^{1}$ \\ Adam Booth, ${ }^{2}$ Peter F. Bove, ${ }^{3}$ Liqiong Gui, ${ }^{1}$ Eric S. White, ${ }^{2}$ and Laura E. Niklason ${ }^{1}$
}

\begin{abstract}
1Departments of Anesthesia and Biomedical Engineering, Yale University, New Haven, Connecticut, USA. 2Internal Medicine, Pulmonary and Critical Care, University of Michigan, Ann Arbor, Michigan, USA. ${ }^{3}$ Cystic Fibrosis/Pulmonary Research Treatment Center,
\end{abstract} University of North Carolina, Chapel Hill, North Carolina, USA.

\begin{abstract}
The use of induced pluripotent stem cells (iPSCs) has been postulated to be the most effective strategy for developing patient-specific respiratory epithelial cells, which may be valuable for lung-related cell therapy and lung tissue engineering. We generated a relatively homogeneous population of alveolar epithelial type II (AETII) and type I (AETI) cells from human iPSCs that had phenotypic properties similar to those of mature human AETII and AETI cells. We used these cells to explore whether lung tissue can be regenerated in vitro. Consistent with an AETII phenotype, we found that up to $\mathbf{9 7 \%}$ of cells were positive for surfactant protein C, $\mathbf{9 5 \%}$ for mucin-1, 93\% for surfactant protein B, and $89 \%$ for the epithelial marker CD54. Additionally, exposing induced AETII to a Wnt/ $\beta$-catenin inhibitor (IWR-1) changed the iPSC-AETII-like phenotype to a predominantly AETI-like phenotype. We found that of induced AET1 cells, more than $90 \%$ were positive for type I markers, $\mathrm{T} 1 \alpha$, and caveolin-1. Acellular lung matrices were prepared from whole rat or human adult lungs treated with decellularization reagents, followed by seeding these matrices with alveolar cells derived from human iPSCs. Under appropriate culture conditions, these progenitor cells adhered to and proliferated within the 3D lung tissue scaffold and displayed markers of differentiated pulmonary epithelium.
\end{abstract}

\section{Introduction}

Lung disease is the third-leading cause of death in the United States, with more than 400,000 deaths annually $(1,2)$. While lung transplantation is a possible treatment for people who have endstage lung disease, it is limited by the low availability of donor lungs; moreover, surgical, medical, and immunological complications cause considerable morbidity and mortality in this population. As a result, many patients die each year while on a waiting list or because of transplant complications $(1,3,4)$.

Transplantation of adult lung stem and progenitor cells or alveolar cells, isolated from human lung, is emerging as an alternative to whole-organ transplantation (5). However, this approach is also limited by the scarcity of human epithelial cells and the difficulties of expanding these cells in vitro. Moreover, the successful engraftment of such cells in vivo in injured lungs has not yet been demonstrated (5-7).

One potential future treatment for severe lung disease is transplantation with engineered lungs that are capable of gas exchange. To avoid immunological rejection, such engineered lungs should be created using individual-specific (autologous) lung and airway cells $(3,8,9)$. Therefore, a substantial emphasis is being placed on identifying a reliable source of functional lung epithelial cells to be used in lung-related therapies $(2,10)$.

Conflict of interest: Laura E. Niklason has a financial interest in Humacyte Inc., a regenerative medicine company. Humacyte did not fund these studies, and Humacyte did not affect the design, interpretation, or reporting of any of the experiments herein.

Note regarding evaluation of this manuscript: Manuscripts authored by scientists associated with Duke University, The University of North Carolina at Chapel Hill, Duke-NUS, and the Sanford-Burnham Medical Research Institute are handled not by members of the editorial board but rather by the science editors, who consult with selected external editors and reviewers.

Citation for this article: J Clin Invest. 2013;123(11):4950-4962. doi:10.1172/JCI68793.
Induced pluripotent stem cells (iPSCs) are the product of adult somatic cell reprogramming to an embryonic-like state by inducing a "forced" expression of specific pluripotent genes $(11,12)$. It is postulated that the use of human iPSCs may be the most effective strategy for developing respiratory epithelial cells that may be valuable in lung-related cell therapies and tissue engineering (13-15). Given that iPSCs can be derived from the patient to be treated, they could provide a cell source that is genetically identical to the patient, allowing tissue generated from these cells to avoid immune rejection $(9,12)$.

The differentiation of human ES cells (ESCs) and iPSCs into pulmonary epithelium has been challenging. Several research groups have reported the successful differentiation toward a range of pulmonary epithelial cell types, including both alveolar type II cells (AETII cells) and other airway epithelium, using a variety of protocols $(1,5,14-19)$. However, conditions for directing hESCs or iPSCs to differentiate along an alveolar epithelial lineage with high homogeneity have not yet been reported, and most protocols generate a mixed population of epithelial cells from hESCs or iPSCs.

Recently, the focus in organ engineering has centered on decellularizing complex organs such as heart, liver, and kidney, and using the acellular matrices as scaffolds for repopulation with organ-specific cells. Because the decellularized organ has the ECM template, it contains appropriate $3 \mathrm{D}$ architecture and regionally specific sites for cellular adhesion $(3,8)$. With ECM derived from donor lungs, the capacity to regenerate lung tissue from autologous cells (e.g., autologous iPSC-derived epithelium) would therefore constitute a major medical advance. One way to accomplish this in lung engineering is to differentiate human iPSCs (iPSCs) into respiratory epithelial cells and/or into putative postnatal stem cells of the respiratory system and to reseed the lung acellular matrix with these cells (9). 
A

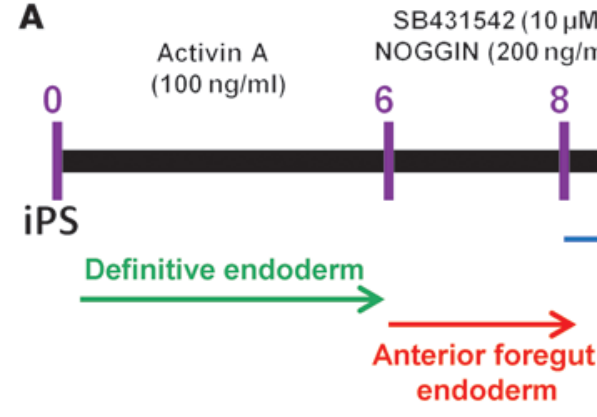

Trophic Factors

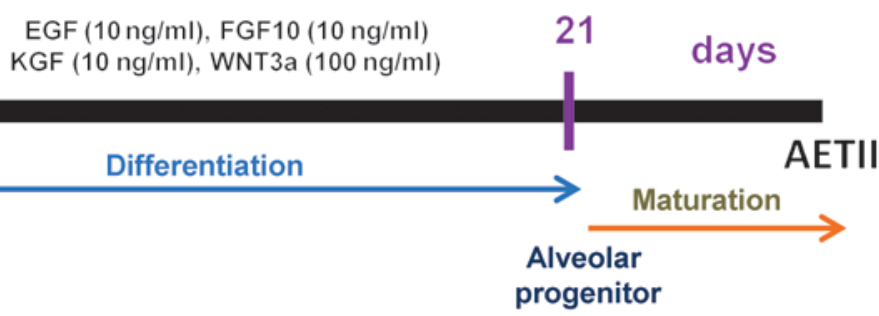

B

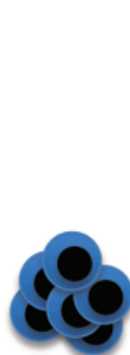

hiPSC

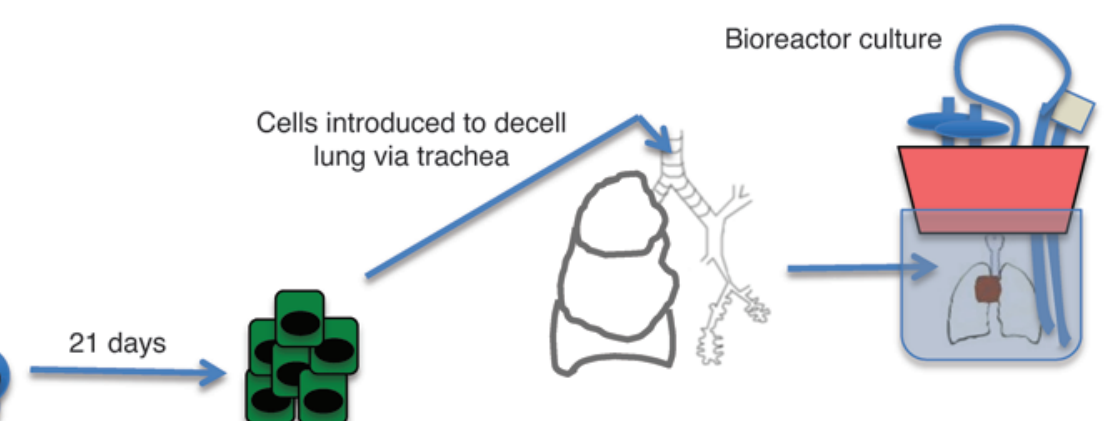

Slice culture

Cells seeded directly onto decell lung slices
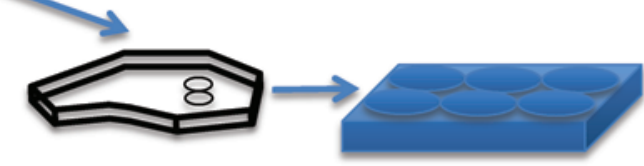

C

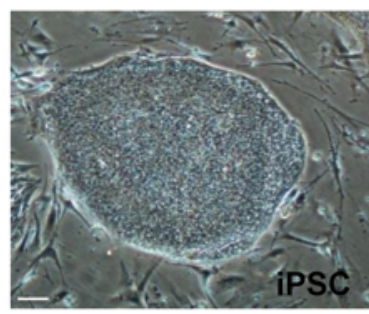

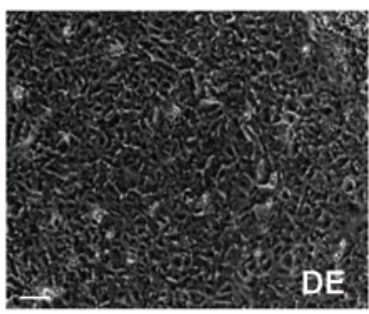

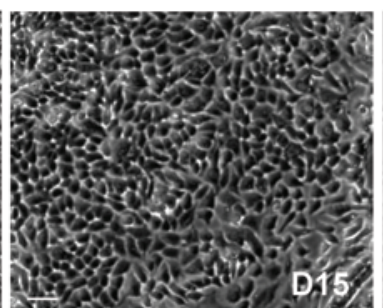

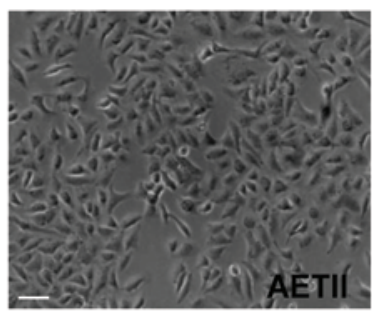

Figure 1

Schematic summarizing the experiment. (A) Schematic protocol for directed differentiation of iPSCs to AETII in vitro in 22 days. Cytokines were added at different steps indicated on top of panel. (B) Schematic summarizing the iPSC differentiation and decellularization-recellularization of both rat and human lung with iPSC-derived AETII cells. decell, decellularized. (C) Phase-contrast images of iPSCs at day 0, DE cells at day 6, and differentiated cells at day 15 and 22, which are termed AETII cells. Scale bars: $63 \mu \mathrm{m}$.

In the present study, we report an efficient and consistent, stepwise differentiation method to generate definitive endoderm (DE), anterior foregut endoderm (AFE), and subsequently, a relatively homogeneous population of human AETII and AETI cells from iPSCs (Figure 1A). These cells not only demonstrate the phenotype of mature hAETI and hAETII cells, but also express a high percentage of type I and II cell markers when compared with freshly isolated hAETI and hAETII cells. Additionally, these iPSC-derived AETII cells are capable of repopulating an acellular lung matrix and give rise to cell types that reside in the distal lung (Table 1).

\section{Results}

Efficient derivation of DE cells. Embryonic lung arises from DE (14, $20,21)$. Therefore, in the first step, we differentiated iPSC from DE by exposing them to saturating concentrations of activin A during the first 6 days of differentiation. iPSC were initially cultured without serum for 48 hours with $100 \mathrm{ng} / \mathrm{ml}$ activin $\mathrm{A}$, and then changed to a low-serum concentration provided with $1 \times \mathrm{B} 27$ culture medium. During the time that iPSCs were exposed to activin A, the majority of the cells in the colonies converted to DE cells, while those cells that did not gradually died, as monitored through visual observation. After 6 days of differentiation, both iPSC clones (denoted as C1 and C2) stained positively for SOX17 and FOXA2, and the majority of the cells were positive for both SOX17 and FOXA2 (Figure 1C, Supplemental Figure 1, A-J, for C1 cells, and Supplemental Figure 2, A-J for C2 cells; supplemental material available online with this article; doi:10.1172/ JCI68793DS1). When we monitored endoderm marker expression using quantitative RT-PCR (qRT-PCR) for SOX17, CXCR4, and FOXA2, no expression of these markers was observed at day 0 ; 


\begin{tabular}{lc}
$\begin{array}{l}\text { Table } 1 \\
\text { Summary of lung development steps and corresponding } \\
\text { markers at each step }\end{array}$ \\
\hline \\
Lung differentiation stages & Markers \\
iPSC & OCT4, SOX2, SSEA4, TRA1-60 \\
DE & CXCR4, SOX17, FOXA2 \\
AFE & NKX2.1, PAX9, TBX1, SOX2 \\
AETII & SPC, SPB, SPA, ICAM-1, NKX2.1 \\
AETI & T1 $\alpha$, caveolin-1, AQ5 \\
\hline
\end{tabular}

expression then increased from day 0 to day 6 in iPSCs exposed to activin A (Supplemental Figure 1L, Supplemental Figure 6A for C1 cells, Supplemental Figure 2L, and Supplemental Figure 6B for $\mathrm{C} 2)$. Flow cytometric analysis demonstrated that the cell population derived from iPSCs at day 6 expressed a high percentage of markers associated with DE, including $92.71 \% \pm 4.0 \%$ for CXCR4, $83.76 \% \pm 2.0 \%$ for SOX 17 , and $87.66 \% \pm 1.2 \%$ for FOXA2 in C1; and $87.23 \% \pm 2.0 \%$ for CXCR $4,91.42 \% \pm 3.0 \%$ for SOX17, and $83.54 \%$ $\pm 1.8 \%$ for FOXA2 in C2 (Supplemental Figure $1 \mathrm{M}$ for C1 cells, and Supplemental Figure 2M for C2). We found our protocol was highly efficient for generating a relatively homogeneous population of DE from iPSCs; based on the dual expression of SOX17 and FOXA2 in iPSC clones, more than $85 \%$ of $\mathrm{C} 1$ and $89 \%$ of $\mathrm{C} 2$ comprised endodermal cells (Supplemental Figure 1K for C1 cells and Supplemental Figure 2K for C2). Both the $\mathrm{C} 1$ clone (which is of fetal lung origin) and the $\mathrm{C} 2$ clone (which is derived from neonatal fibroblasts) yielded similar results. This suggests that this protocol may be generalized to other iPSC lines from other cell origins or that are reprogrammed using other techniques.

Generating AFE from DE cells. Following developmental paradigms, directed differentiation of iPSCs to alveolar epithelium should proceed by generation of $\mathrm{DE}$, followed by patterning into AFE. Differentiation of AFE cells from DE was induced by exposing cells to NOGGIN (200 ng/ml) and SB-431524 (10 mM) for 2 days per the conditions described previously by Green and colleagues $(1,14,15)$. Application of NOGGIN/SB-431542 to DE yielded a highly enriched population of cells with strong expression of markers associated with the AFE phenotype, including SOX2, PAX9, and TBX1 in both clones. The majority of cells coexpressed SOX2 and FOXA2, as demonstrated by immunostaining at day 8 (Figure 2, A-J for C1 cells, and Supplemental Figure 3, A-J for C2). Cells that were negative for DE markers gradually died off after switching to AFE differentiation medium, as visualized by microscopy. In addition, our results show that inhibition of TGF- $\beta$ signaling and activin $A /$ nodal signaling with NOGGIN/SB-431542 was sufficient in the 2 iPSC clones tested to increase the anterior endoderm cell population (as defined by $\mathrm{FOXA} 2{ }^{+} \mathrm{SOX}_{2}^{+}$) up to $92 \%-95 \%$ as compared with less than $0.1 \%$ without NOGGIN/SB-431542 (Figure $2 \mathrm{~K}$ for C1 cells, and Supplemental Figure $3 \mathrm{~K}$ for C2). qRT-PCR revealed a relatively modest increase in both $P A X 9$ and TBX1 when compared with expression of $S O X 2$, which was highly expressed in AFE cells derived from both iPSC colonies at day 8 (Figure 2L for C1 cells, and Supplemental Figure 3L for C2). After activin A removal at day 6 and switching to NOGGIN/SB-431542, we observed that both CXCR4 and SOX17 decreased from day 6 to day 12. In the case of FOXA2, we observed an increase in expression at day 8 , followed by a decrease with time in culture (Supplemental Figure 6A for C1 cells, and Supplemental Figure 6B for C2). These results are expected, given that endoderm is a transient stage in lung development and is expected to peak and then fade as stem cells differentiate toward later phenotypes (22).

Prior to lung differentiation, all cells that will belong to the pulmonary lineage must first progress through a primordial progenitor stage defined by the upregulation of the ventral marker NKX2.1 (1, 16). NKX2.1 (homeodomain-containing transcription factor) is the earliest known marker associated with commitment to thyroid and lung, but several studies suggest that NKX2.1 induction is indicative of commitment to a lung, rather than a thyroid fate $(1,14,16)$. Replacing activin A with NOGGIN/SB-431542 from day 6 to day 8 , followed by the addition of a cocktail containing BMP4/Wnt3a/bFGF/KGF, induced NKX2.1 expression in the AFE cell population at day 13. Most of the NKX2.1-positive cells in both iPSC clones costained with the endoderm marker FOXA2 (Figure 2, M and N, for C1 cells, and Supplemental Figure 3, M and $\mathrm{N}$ for $\mathrm{C} 2$ ). Additionally, flow cytometric analysis showed that $24 \% \pm 2 \%$ of the AFE cell population derived from C1 and $26 \% \pm 3 \%$ from C2 was positive for NKX2.1, as compared with less than $1 \%$ in activin A-induced cells and in cells cultured in medium without NOGGIN/SB-431542 at day 13. Continuous activin A treatment from day 6 to day 8 without the addition of NOGGIN/SB-431542 resulted in rare FOXA2 ${ }^{+} \mathrm{SOX} 2^{+}$cells and few $\mathrm{NKX} 2.1^{+}$cells (Figure $2 \mathrm{O}$ for $\mathrm{C} 1$ cells, and Supplemental Figure $3 \mathrm{O}$ for C2). Collectively, these expression data show that in activin $\mathrm{A}$-induced DE, exposure to NOGGIN/SB-431542 results in a highly enriched population of cells with an AFE phenotype.

ECM protein effects on differentiation. In traditional stem cell cultivation/differentiation experiments, growth factors (GFs) are added in soluble form in order to provide signals for tissuespecific differentiation $(14,15,17,18,20)$. However, differentiation has recently become increasingly linked to mechanobiological concepts such as interaction between cells and the ECM (23-25). Moreover, during development, ECM protein expression represents some of the most important inducers of organ fate. Accordingly, before switching medium to NOGGIN/SB-431542 to generate AFE from human pluripotent cells, the DE cells were split with trypsin and reseeded at a ratio of 1:1-2 on ECM-coated plates in medium containing NOGGIN/SB-431542 for 48 hours. We tested adhesion of DE cells to fibronectin, collagen I, collagen IV, Matrigel, and a mixture of human ECM proteins (consisting of collagens, laminin, fibronectin, tenascin, elastin, and a number of proteoglycans and glycosaminoglycans; Sigma-Aldrich). Fibronectin, collagen I, collagen IV, and laminin are principal components of lung matrix, and DE cells attach well to all of these proteins. However, mixed human ECM protein resulted in faster DE cell attachment and significantly higher expression of $S P C, S P B$, and $N K X 2.1$ genes on both day 15 and day 30 (Supplemental Figure 4, A-C).

Efficient derivation of purified lung AETII from AFE. After differentiation to AFE on day 8, the medium was switched to alveolar differentiation medium containing FGF-10, EGF, WNT3a, KGF, and RA for 14 days on human ECM protein. These factors or reagents were chosen through empiric studies and are thought to play a crucial role in alveolar pneumocyte differentiation and lung development $(1,14,15,17,18,20)$. Compared with other reports, we found that the differentiation cocktail that lacked BMP4 in the final stage resulted in distal markers, especially those associated 

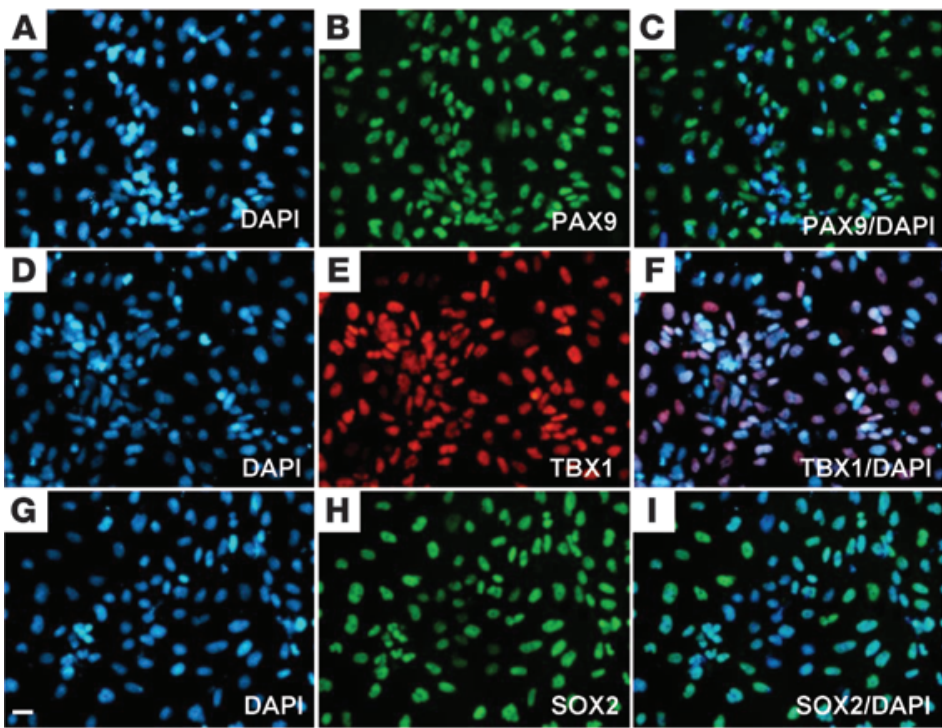

$\mathbf{M}$
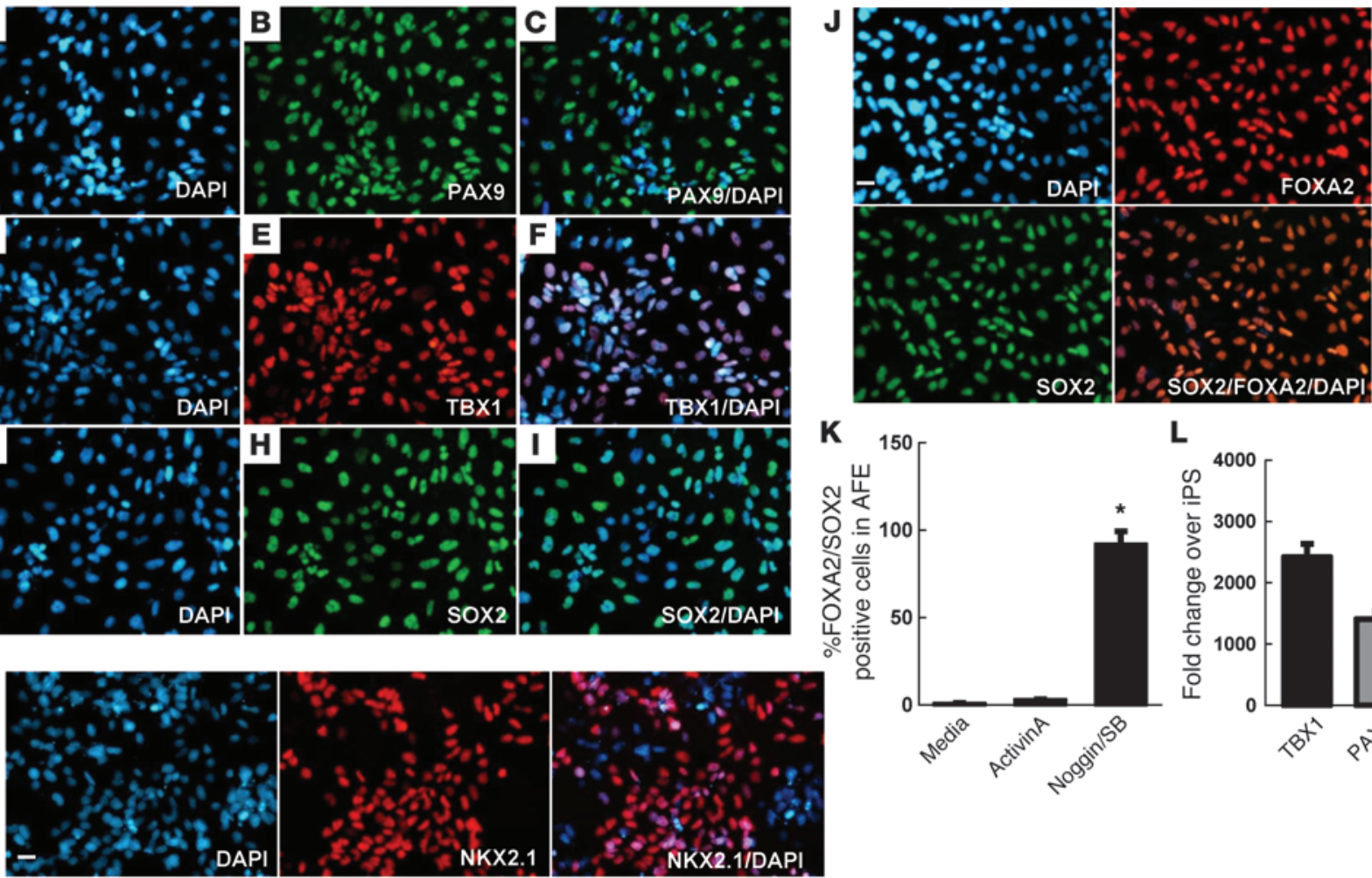

$\mathbf{L}$
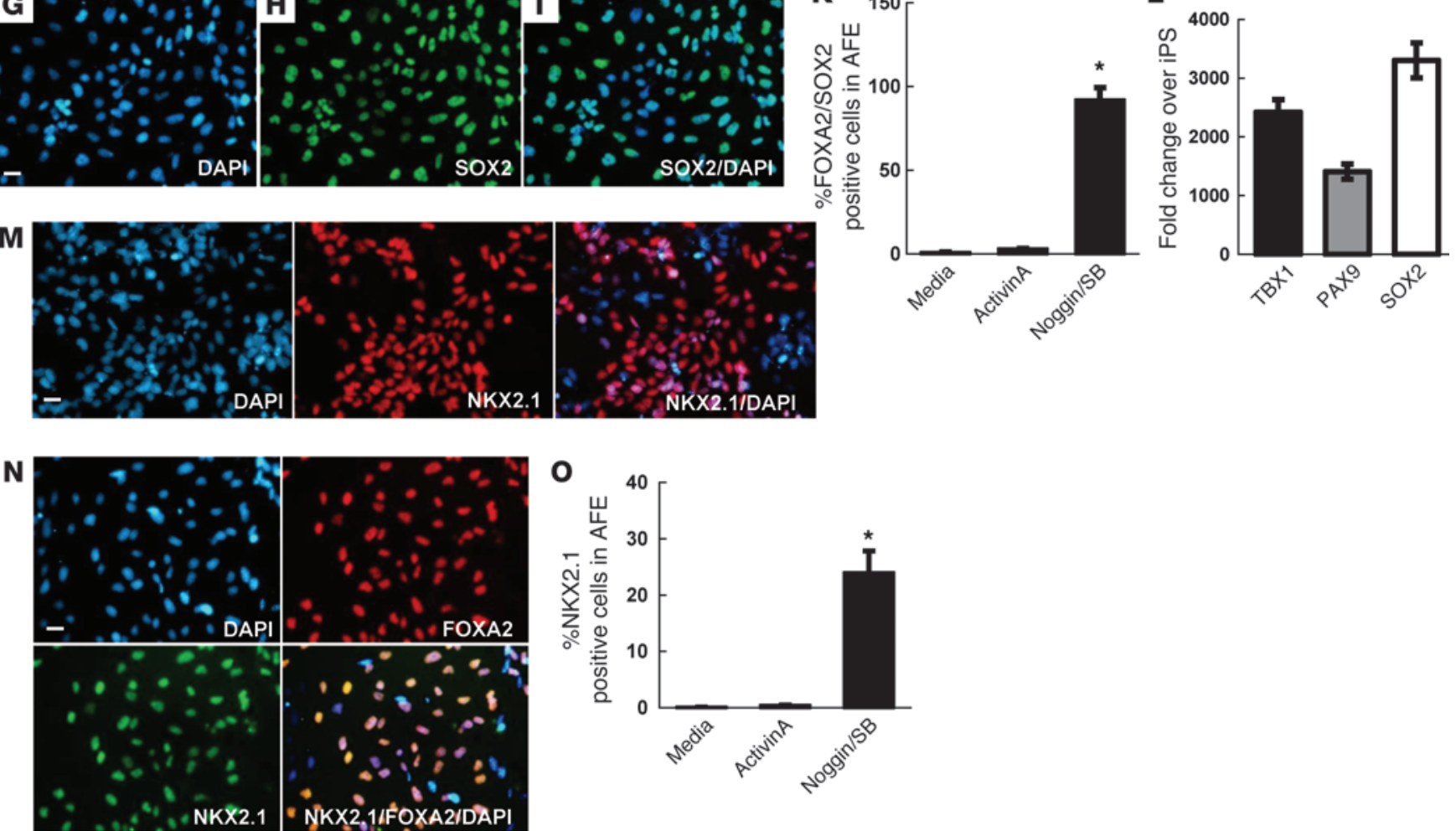

Figure 2

Characterization of cells at day 8 of differentiation to produce AFE. (A-I) Immunofluorescence analysis of AFE markers. (A, D, and G) DAPI staining for nuclei; (B, E, and H) PAX9-, TBX1- and SOX2-positive cells. (C, F, and I) Merge at day 8. (J) Immunofluorescence staining showing AFE cells are positive for both SOX2 and FOXA2. (K) Flow cytometric analysis of double-positive cells for SOX2 and FOXA2 in AFE cells at day 8 compared with cells cultured in activin A and RPMI medium only ( $y$ axis: percentage of positive cells for FOXA2/SOX2 ). (L) mRNA expression of SOX2, TBX1 and PAX9 in AFE generated from DE cells in vitro at day 8 (data expressed as quantification of mRNA normalized to GAPDH and average fold change in gene expression over iPSCs; $y$ axis, fold changes in gene expression compared with iPSC). (M) Expression of NKX2.1 on day 13 after induction AFE quantified by DAPI staining for nuclei, NKX2.1 positive cells, and merge. (N) Immunofluorescence staining showing NKX2.1 cells in AFE stained positive for FOXA2, indicating these cells are more lung progenitor rather than thyroid progenitor. (0) Flow cytometric analysis of positive cells for NKX2.1. Up to 24\% of AFE cells were positive for NKX2.1 at day 13. ( $y$ axis, percentages of cells positive for NKX2.1). Bars indicate mean \pm SEM of $n=3$ independent experiments for qRT-PCR and flow cytometry. ${ }^{*} P<0.05$. Scale bars: $31 \mu \mathrm{m}$.

with type II pneumocytes. After day 22, the cells - now termed AETII cells (Figure 1, A and B) - were maintained in SAGM culture medium containing $1 \%$ FBS. AETII cells derived from both the $\mathrm{C} 1$ and $\mathrm{C} 2$ clones were strongly positive for type II markers, including pro-SPC, pro-surfactant protein B (pro-SPB), mucin-1, and surfactant protein A (SPA). In addition to the positive marker expression associated with type II cells, we also assayed for the presence of lamellar bodies, typical of human type II cells, by elec- tron microscopy in the iPSC-derived AETII cells. TEM identification of lamellar bodies is used as a method for positively identifying type 2 pneumocytes. The TEM data clearly show the presence of lamellar bodies in the iPSC-derived AETII cells (Figure 3, A-F for C1 cells, and Supplemental Figure 5, A-F for C2). qRT-PCR demonstrated a high percentage of expression of type II cell markers in iPSC-AETII cells that was comparable to expression levels of freshly isolated human primary ATII cells (hATII cells) (Figure 
$3 \mathrm{G}$ for C1 cells, and Supplemental Figure 5G for C2). Up to $97 \%$ of cells were positive for SPC, $92 \% \pm 0.9 \%$ positive for mucin- $1,89 \%$ $\pm 0.9 \%$ positive for SPB, and the vast majority of the cells, $94 \% \pm$ $0.9 \%$, expressed the epithelial surface marker CD54 in clone C1 and $96.27 \% \pm 0.5 \%$ for SPC, $94.42 \% \pm 0.3 \%$ for SPB and $91.54 \% \pm$ $1.8 \%$ for mucin- 1 and $89.83 \% \pm 0.5 \%$ for CD54 in clone C2. Moreover, AETII cells were negative for CCSP (a Clara cell marker), p63 (basal stem cell marker), and SOX2 (proximal airway epithelial cell marker) by FACS analysis, indicating that these cells are a relatively homogeneous population of type II cells (Figure 3, H and I for C1 cells, and Supplemental Figure 5, H and I for C2). ELISA measurements of SPC protein in the cell culture supernatants indicated that day 13 cells synthesized and secreted SPC at a rate of 16.78 $\mathrm{ng} / \mathrm{ml}$ and $15.78 \mathrm{ng} / \mathrm{ml}$ in C1 and C2 clones, respectively, every 24 hours; the rate of secretion was significantly increased on day 22 of differentiation when compared with secretion at day $13(P<0.05)$. At day 22, the iPSC-AETII cells from C1 and C2 produced $68.5 \mathrm{ng} /$ $\mathrm{ml}$ and $71.5 \mathrm{ng} / \mathrm{ml} \mathrm{SPC}$ every 24 hours, which was comparable to that produced by freshly isolated human AETII cells $(82.95 \mathrm{ng} / \mathrm{ml})$ (Figure 3J for C1 cells, and Supplemental Figure 5J for C2).

A previous study has found that airway progenitor cells, which ultimately give rise to trachea, bronchus and bronchioles, are $\mathrm{NKX} 2.1^{+} \mathrm{SOX} 2^{+}$and sustain high levels of SOX2 expression (15). However, by day 22 , no NKX $2.1^{+} \mathrm{SOX} 2^{+}$cells or single-positive $\mathrm{SOX}^{+}$cells were detected in the population of differentiated iPSC-AETII cells. All the AETII cells were also negative for CCSP and $\mathrm{p} 63$ as determined by flow cytometry (Figure $3 \mathrm{H}$ for $\mathrm{C} 1$ cells, and Supplemental Figure 5H for C2 cells). This may indicate that these cells are not airway progenitor cells. Since AFE can be theoretically differentiated into cells expressing markers of thyroid, parathyroid, and lung (1), we checked the expression of CD31 (endothelial marker), albumin (mature hepatocyte marker), and TSGHR (thyroid cell marker) by qRT-PCR to determine whether the iPSC-AETII cells were contaminated by cells from these other lineages. No specific markers of thyroid, endothelial cells, or hepatocyte lineage were detected in iPSC-AETII cells at day 22, thereby confirming the absence of cells from these lineages in the differentiated lung progenitor population (Figure 3I for C1 cells, and Supplemental Figure 5I for C2 cells).

The expression of AFE markers (SOX2, PAX9, and TBX1) and DE markers (SOX17, CXCR4, and FOXA2) decreased from day 8 to day 22. By day 32, none of these markers were detectable in iPSC-AETII cells. In the case of FOXA2 and SOX2, after activin A removal at day 6 and switching to NOGGIN/SB-431542, we observed an increase in FOXA2 and SOX2 expression at day 8 followed by a decrease in the expression of these genes in culture over time (Supplemental Figure 6, A and C, Supplemental Figure 10B for C1 cells, Supplemental Figure 6, B and D, and Supplemental Figure 10C for C2 cells).

Following exposure to alveolar pneumocyte induction medium containing Wnt3a, EGF, KGF, and FGF at day 9 of culture, there was lower expression of AFE genes, especially SOX2, and there was a concomitant upregulation of NKX2.1. After day 8, SOX2 was rapidly downregulated while NKX2.1 was upregulated. The expression of NKX2.1 gradually increased from $24 \% \pm 2 \%$ at day 13 to $94 \%$ $\pm 0.4 \%$ at days $20-22$ in C1. qRT-PCR and flow cytometry revealed that the percentage of SPC-positive cells gradually increased from $58 \% \pm 1.6 \%$ at day 13 to $90 \% \pm 0.4 \%$ at days $20-22$ during differentiation in the C1 clone. The same pattern of NKX2.1 and SPC expression was observed in the $\mathrm{C} 2$ clone during differentiation from day 8 to day 22 (Supplemental Figure 7, A-D for C1 cells, and Supplemental Figure 8, A-D for C2 cells). Between days 8 and $22, \mathrm{NKX} 2.1^{+}$cells proliferated slowly, ultimately leading to an increase in the number of NKX2.1- and SPC-positive cells. The amount of cell death, following the switch in culture medium to alveolar epithelium differentiation medium, was negligible when compared to the earlier medium switch from DE to AFE differentiation medium from day 0 to day 8 . Immunostaining for NKX2.1 ${ }^{+}$ cells at different days (day 13, 18, and 21) demonstrated that colonies expressing NKX2.1 were gradually expanded and overgrew the NKX2.1 negative cells. This ultimately led to an increase in the number of NKX2.1-positive cells, as shown by a gradual progression from $24 \%$ at day 13 to $94 \%$ at days $20-22$.

Since several recent studies have reported CD166 and $\alpha_{6} \beta_{4}$ to be markers of lung epithelial progenitors (26-29), we characterized the expression of these markers during the differentiation of iPSC to epithelial cells at several time points. Flow cytometry revealed that the percentage of CD166-positive cells gradually increased from $15 \% \pm 0.8 \%$ at day 13 to $35 \% \pm 1.4 \%$ at days $20-22$ during differentiation in the $\mathrm{C} 1$ clone. The expression of CD166 subsequently decreased in mature cultures of iPSC-derived epithelial cells at day 28. In the case of $\alpha_{6} \beta_{4}$, the expression progressively increased from $10 \% \pm 0.7 \%$ at day 13 to $25 \% \pm 1.2 \%$ at day 18 in C1. By day 22 only $7 \%-8 \%$ of the cells were positive for $\alpha_{6} \beta_{4}$. The same pattern of CD166 and $\alpha_{6} \beta_{4}$ expression was observed in the $\mathrm{C} 2$ clone during differentiation from day 8 to day 22 (Supplemental Figure 9, A and B for C1 cells, and Supplemental Figure 9, C and D for C2 cells).

Replacing the NOGGIN/SB-431542 medium with differentiation medium at day 9 of culture resulted in decreasing expression of pluripotency genes such as OCT4 and Nanog over time. As expected, from day 13 to day 22, the pluripotency gene expression was almost undetectable. Flow cytometry on iPSC-derived AETII cells from both clones showed that SPC-positive cells at day 22 were negative for OCT4 (Supplemental Figure 10, A-E for C1 and C2 cells).

Since type II cells can spontaneously differentiate into cells expressing markers of type I cells (30-32). we assessed the expression of type I markers T1 $\alpha$, aquaporin-5 (AQ5), and caveolin- 1 by flow cytometry and qPCR. Flow cytometry revealed that $8 \%-11 \%$ of cells expressed type I markers following the differentiation protocol. Up to $9 \%$ of cells were positive for AQ5, 9.6\% positive for caveolin-1, and $8.1 \%$ expressed the type I surface marker T1 $\alpha$ in clone C1 (Figure 4C).

Differentiation of iPSC-derived AETII to type I cells. To further differentiate iPSC-AETII to AETI, we next examined the effect of modulating Wnt $/ \beta$-catenin signaling on type I marker expression in AETII cells. We determined whether the selective $\mathrm{Wnt} / \beta$-catenin inhibitor IWR-1 $(20,30)$ would modulate the differentiation of iPSC-AETII cells to AETI cells. Differentiated AETII cells at day 22 were cultured on human ECM protein-coated plates in DMEM-10\% FBS supplemented with $100 \mathrm{mM}$ IWR-1 for 7 days. As data from independent experiments indicated, incubation of iPSC-AETII cells with IWR-1 induced the differentiation of AETII cells to the AETI cell phenotype (Figure 4, A-D). Following treatment with IWR-1, there was a significant increase in AETI markers AQ5, T1 $\alpha$, and caveolin-1 in iPSC-derived AETII compared with untreated AETII, as determined by immunostaining (Figure 4, A and B). Flow cytometry revealed (as shown in Figure 4C) that up to 92\% of cells were positive for AQ5, 98\% positive for caveolin-1, and $88 \%$ of the cells expressed the epithelial marker T1 $\alpha$. In contrast, the AETII cell marker SPC 
iPSC-AETII
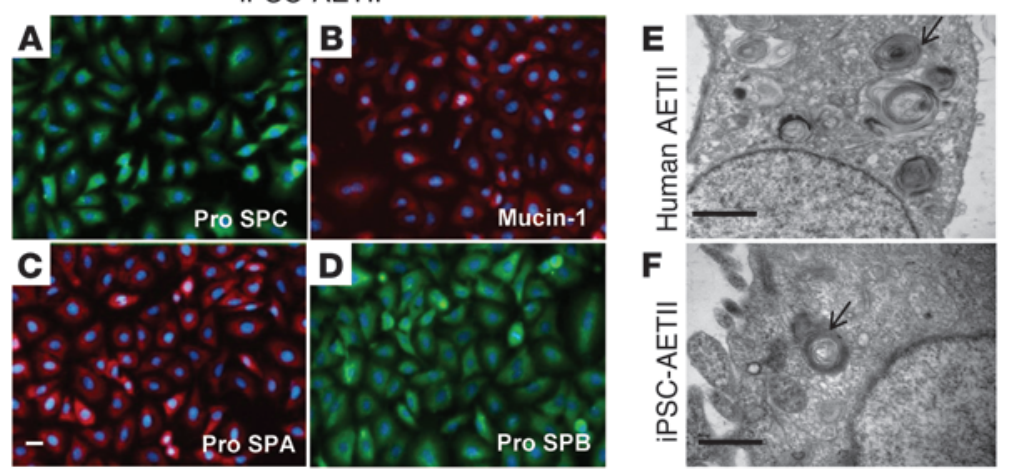

G
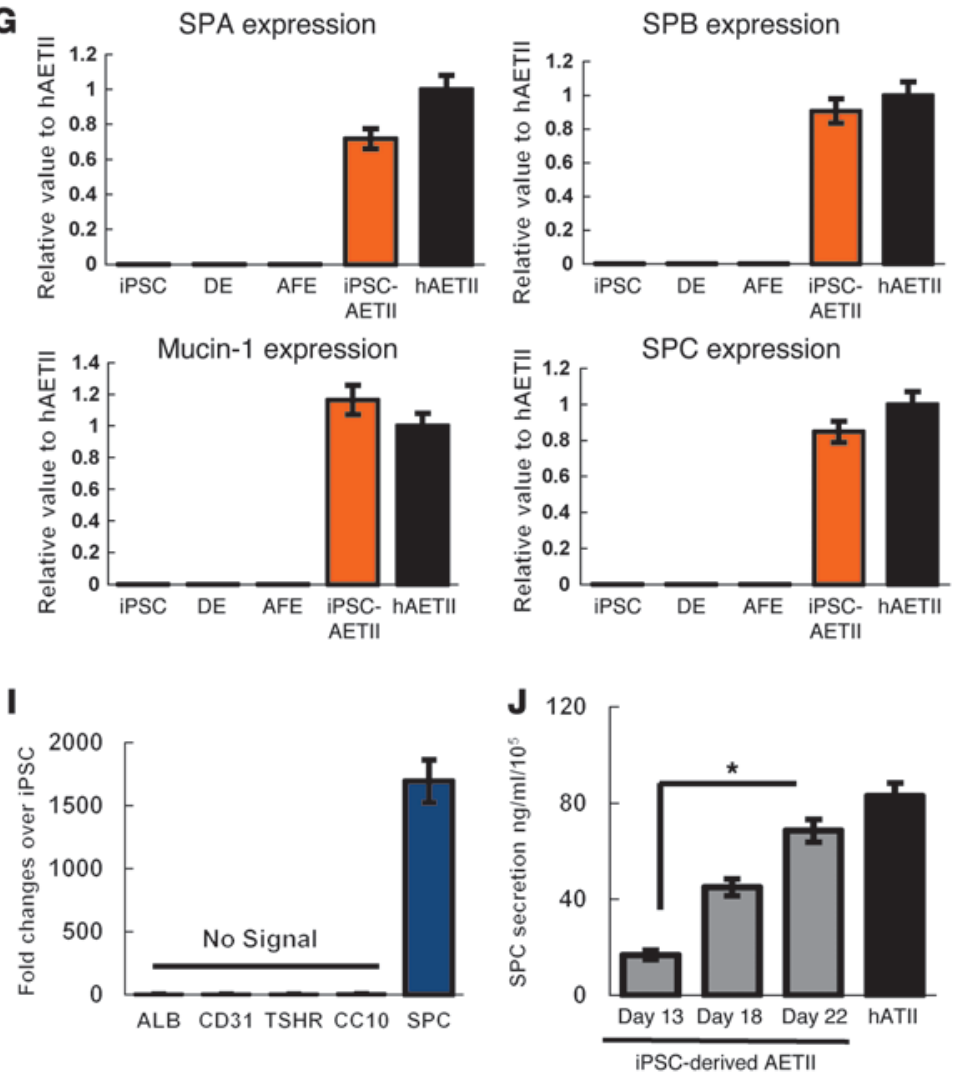

$\mathbf{H}$
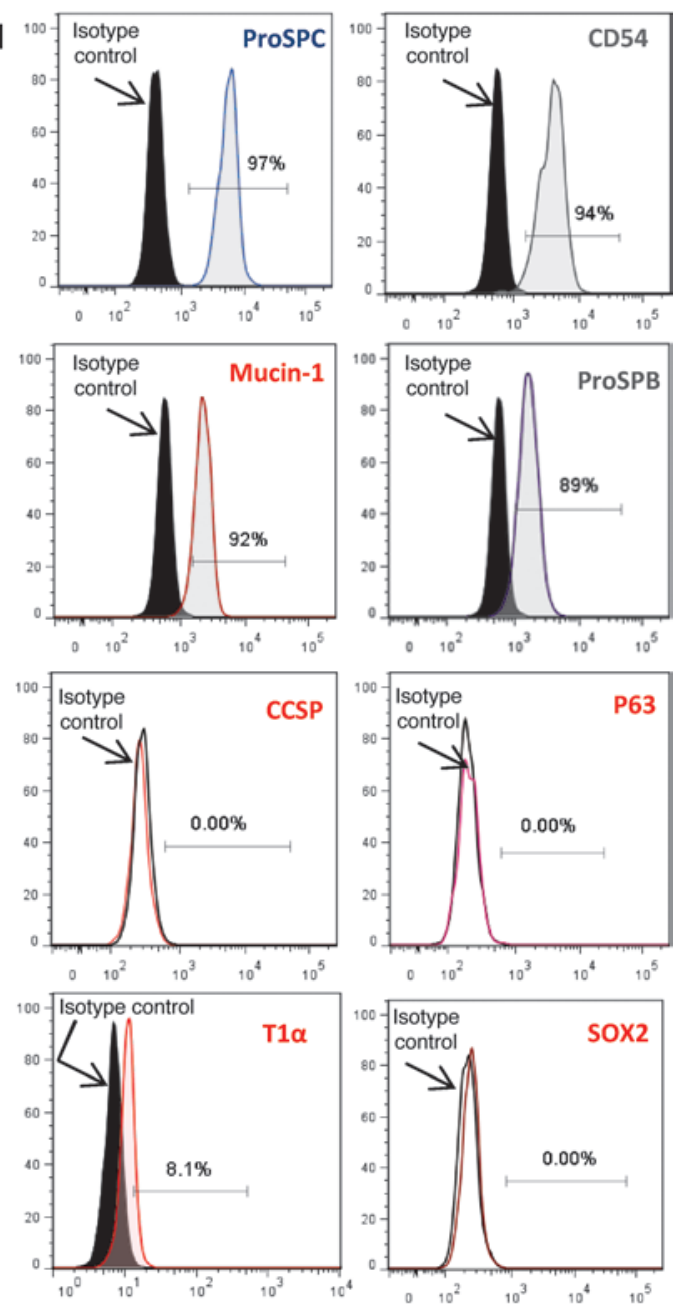

$\mathbf{K}$

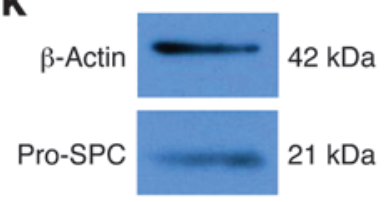

\section{Figure 3}

Functional characterization of AETII cells derived from iPSCs, day 22 of differentiation (C1 clone). (A-D) Immunostaining of AETII marker: (A) pro-SPC, (B) mucin-1, (C) pro-SPA, (D) pro-SPB. Scale bar: $63 \mu \mathrm{m}$. (E and F) Transmission electron microscopy represents (E) human AETII and (F) iPSC-derived AETII containing characteristic cytoplasmic laminar bodies. Scale bars: $0.5 \mu \mathrm{m}$. (G) qRT-PCR analysis in undifferentiated iPSC, $\mathrm{DE}, \mathrm{AFE}$, and differentiated AETII cells compared with human AETII from 3 independent experiments. Values from the triplicate PCR reactions for a GOI (SPA, SPB, SPC, and mucin-1) were normalized against average GAPDH Ct values from the same cDNA sample. Fold change of GOI tra-script levels between iPS-derived AETII and human type II cells equals $2^{-\Delta \Delta C t}$, where $\Delta \mathrm{Ct}=\mathrm{Ct}_{(\mathrm{GOI})}-\mathrm{Ct}_{(\mathrm{GAPDH})}$, and $\Delta \Delta \mathrm{Ct}=\Delta \mathrm{Ct}_{(\mathrm{AETIII})}-\Delta \mathrm{Ct}_{(\mathrm{ATII})}$. (H) Flow cytometry analysis for the percentage of positive cells for AETII and AETI markers at day 22. Cells were negative for p63 and SOX2. (I) Expression of albumin, CD31, TSHR, and CC10 (CCSP) in iPSC-AETII. Cells were negative for genes indicative of other lineages at day 22. (J) Amount of secreted SPC in the iPSC-derived AETII supernatants collected during the time course of differentiation compared with human type II cells determined by ELISA. (K) Western blot for pro-SPC in iPSC-AETII at day 22 and $\beta$-actin as an internal control. Bars indicate \pm SEM and $n=3$ independent experiments for qRT-PCR, ELISA, and flow cytometry. ${ }^{*} P<0.05$.

decreased significantly, as determined by flow cytometry (Figure 4C). qRT-PCR demonstrated a high percentage of expression of type I cell markers in iPSC-AETII cells exposed to IWR-1 that was comparable to expression levels of freshly isolated hAETI cells (Figure 4D)
Repopulation of rat and human acellular matrix with iPSC-derived AETII. To explore the regenerative potential of iPS-derived AETII cells to generate lung tissue in vitro, lungs from adult humans and rats were decellularized by processes that remove cellular 

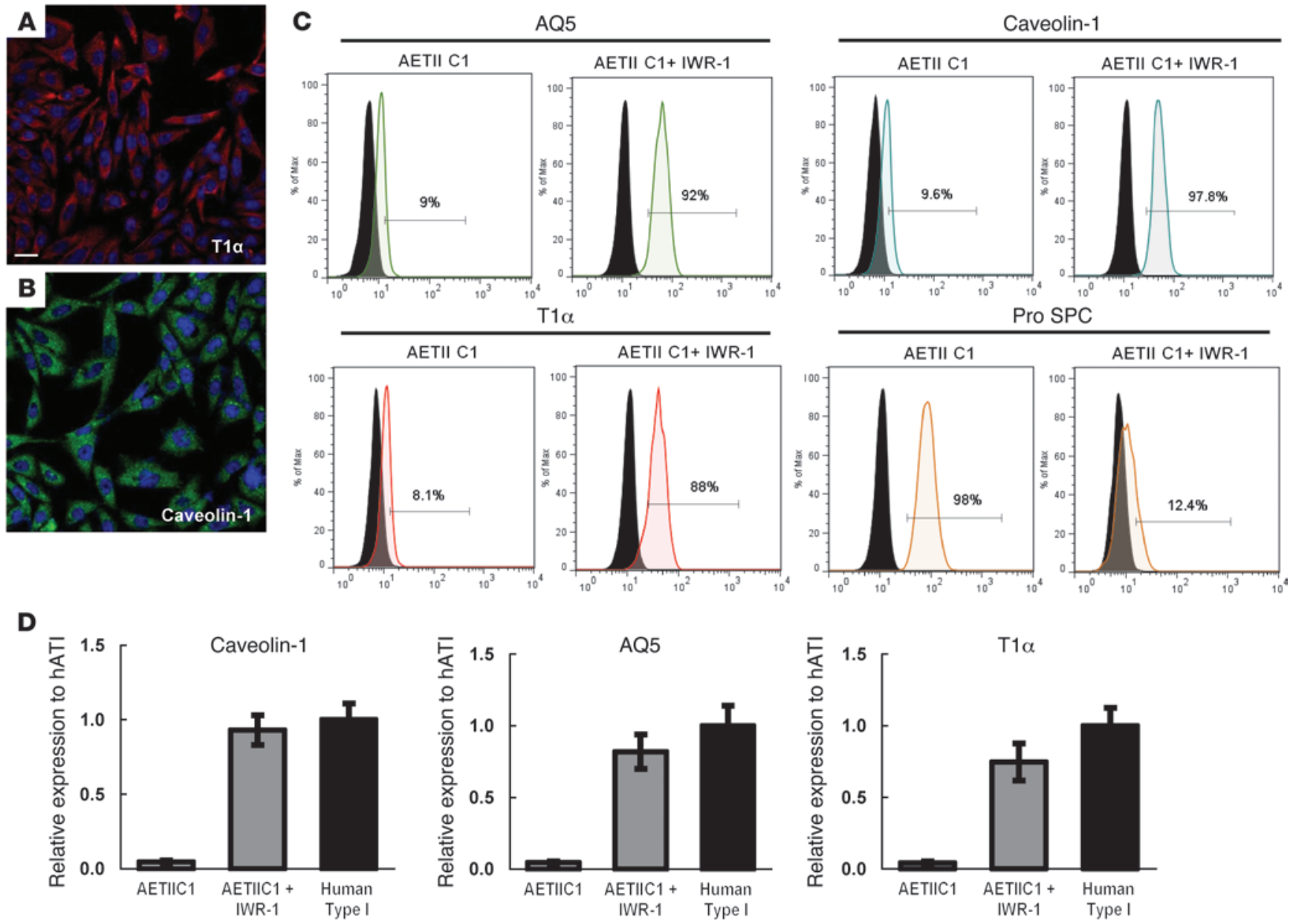

Figure 4

Functional characterization of AETI cells derived from iPSCs, day 29 of differentiation (C1 clone). (A and B) Immunofluorescent staining of alveolar type I marker (A) T1 $\alpha$ (B) caveolin-1. Scale bar: $63 \mu \mathrm{m}$. (C) Flow cytometry analysis for the percentage of positive cells for alveolar type I marker at day 29 in the presence and absence of IWR-1. ( $y$ axis, percentage of positive cells). (D) qRT-PCR analysis in AETI cells as compared with native human type I (AETI) cells, from 3 independent experiments. Values from the triplicate PCR reactions for a GOI $(A Q 5, T 1 \alpha$, caveolin-1) were normalized against average GAPDH Ct values from the same cDNA sample. Fold change of GOI transcript levels between iPS-derived AETI and human type I cells equals $2^{-\Delta \Delta \mathrm{Ct}}$, where $\Delta \mathrm{Ct}=\mathrm{Ct}_{(\mathrm{GOI})}-\mathrm{Ct}_{(\mathrm{GAPDH})}$ and $\Delta \Delta \mathrm{Ct}=\Delta \mathrm{Ct}_{(\mathrm{AETI})}-\Delta \mathrm{Ct}(\mathrm{hAETI})$. $(y$ axis, relative gene expression compared with human type I cells). Bars indicate \pm SEM and $n=3$ independent experiments for qRT-PCR, ELISA, and flow cytometry.

components but leave behind a scaffold of ECM that retains the hierarchical branching structures of airways and vasculature $(8,33)$. This is an assay that was recently developed to test the regenerative potential of primary lung epithelial cells or stem cell-derived lung epithelial cells $(1,8,34,35)$. Both ATI and ATII pneumocytes are differentiated cell types; however, several reports have demonstrated that ATII cells retain a level of plasticity. When damage occurs to the ATI pneumocytes, ATII cells proliferate and can, in turn, differentiate into ATI cells $(29,30$, 32). Therefore, in this study, we explored the capacity of iPSCderived type II cells to repopulate the airway compartment of a decellularized lung ECM. We utilized iPSC-AETII cells at day 28 to repopulate the acellular matrices. This stage seemed most suitable because of their commitment to a pneumocyte type II phenotype and function. We then cultured the seeded matrix in a bioreactor that was designed and described previously $(8,36)$. This bioreactor is capable of replicating key aspects of in vivo fetal lung environment, including vascular perfusion and liquid ventilation. In addition to seeding iPSC-AETII cells into decellularized whole rat lung tissue, we also seeded iPSC-AETII cells onto slices of either rat or human acellular lung matrix cultured in 6-well plates (Figure 1B).

For the rat lung bioreactor experiments, approximately $40 \times 10^{6}$ cells were injected into the airway through the trachea to repopulate the decellularized rat lung matrix (Figure 1B). The seeded matrix was then cultured and maintained for up to 7 days in a bioreactor in SAGM (1\% FBS). H\&E staining showed that iPSCAETII cells were able to diffusely repopulate alveolar lung structures within distal lung. The majority of seeded AETII cells still showed approximate AETII morphology - a cuboidal shape and round single nuclei (Figure 5, A-C for C1 cells, and Supplemental Figure 11, A-C for C2 cells). Many AETII cells within the lung matrix expressed the type II cell marker, pro-SPC, and NKX2.1. In native lung, this marker is normally found on AETII cells. In the 
Decellularized lung
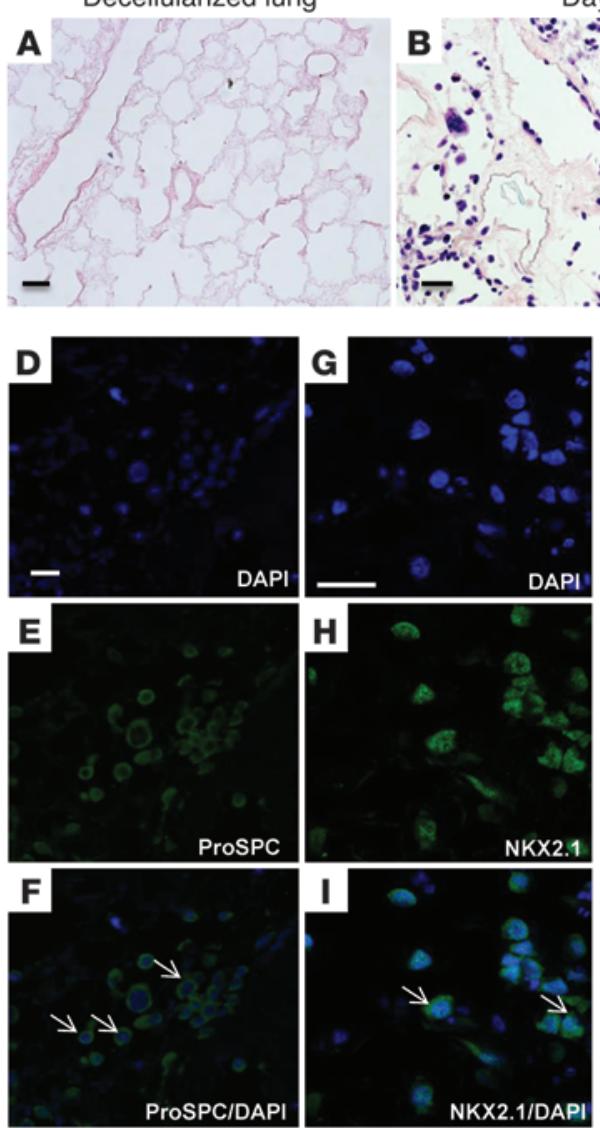

$\mathbf{P}$

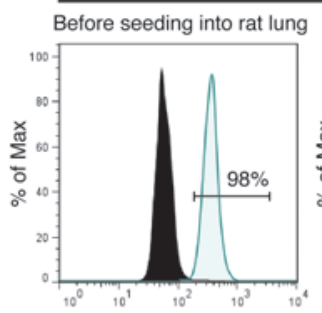

ProSPC
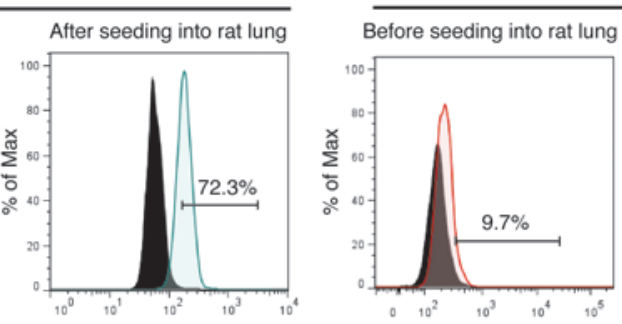

After seeding into rat lung
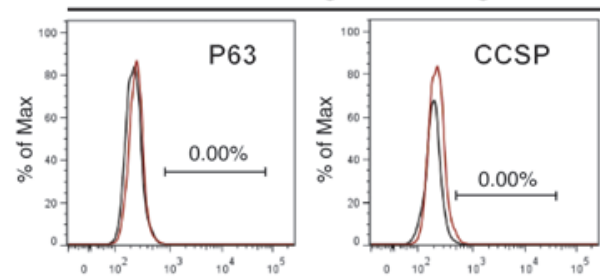

$\mathrm{T} 1 \alpha$
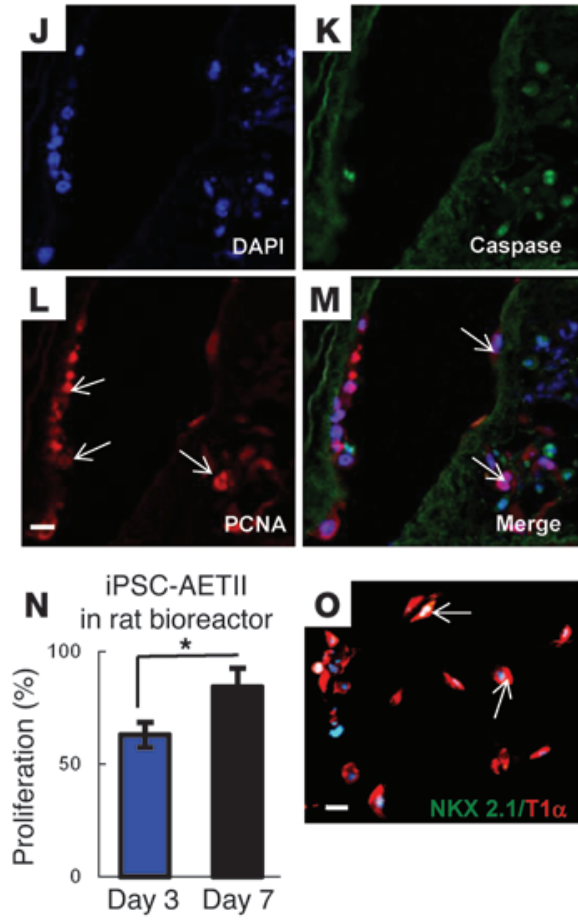

Day 7
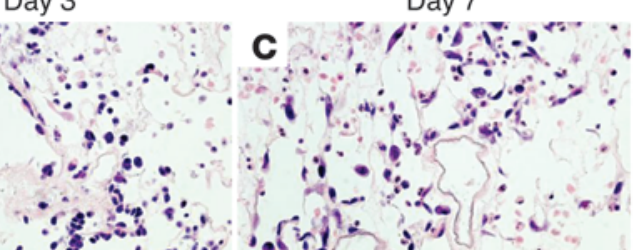

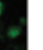

Figure 5

iPSC-derived AETII recellularized $3 \mathrm{D}$ rat lung tissue scaffolds in a bioreactor. (A) H\&E staining of decellularized rat lung; (B and C) H\&E staining of 3- and 7-day seeded rat lung with iPSC-derived AETII cells cultured in a bioreactor. Scale bars: 25 um. (D-F) Immunofluorescent staining for pro-SPC in AETII seeded cells at day 3. (D) DAPI staining; (E) pro-SPC; (F) merge (arrows in $\mathbf{F}$ indicate cells positive for pro-SPC). (G-I) Immunostaining for NKX2.1 at day 7. (G) DAPI, (H) NKX2.1, (I) merge (arrows in $5 \mathrm{I}$ indicate cells positive for NKX2.1) (J-M) Caspase and PCNA immunostaining at day 7 (arrows indicates cells positive for PCNA in $\mathbf{L}$ and caspase in M). Scale bar: $25 \mu \mathrm{m}$. (N) Proliferation at day 7 compared with day 3. iPSC-AETII displayed a significantly increased fractional proliferation $(P<0.05)$ after 7 days when they were stained for PCNA ( $y$ axis, percentage proliferation based on the number of positive nuclei stained for PCNA) (O) Immunostaining of the few engrafted epithelial cells that acquired flattened morphology, positive for $T 1 \alpha$, and negative for NKX2.1 at day 7. Scale bar: $63 \mu \mathrm{m}$. Arrows in $\mathbf{O}$ indicate cells positive for $\mathrm{T} 1 \alpha$. (P) Flow cytometry for SPC, $\mathrm{T} 1 \alpha$, CCSP, p63, and SOX2 before and after seeding into rat lung scaffold in bioreactor. The number of SPC-positive cells decreased during 7-day culture, while the number of cells positive for $\mathrm{T} 1 \alpha$ increased from $9 \%$ to $31.2 \%$. All differentiated cells from iPSCs were negative for CCSP, p63, and SOX2 before and after cell seeding. reseeded rat lungs, cellular expression of pro-SPC was robust in the alveoli by day 3 and remained present at day 7 (Figure 5, D-I for C1 cells, and Supplemental Figure 11, D-I for C2 cells).

We investigated whether iPSC-derived AETII are able to proliferate in the acellular matrix. After culturing iPSC-AETII cells in the bioreactor, sections from the reseeded lung at day 3 and day 7 were stained for proliferating cell nuclear antigen (PCNA) expression. The majority of cells on the rat scaffold expressed PCNA at both day 3 and day 7 while they displayed few markers of apoptotic cell death, as determined by immunostaining for caspase-3. However, iPSC-AETII cultured in the rat lung scaffold for 7 days had an increased rate of positive PCNA staining 
when compared with the day-3 cultures. The data suggest that the iPSC-AETII are able to proliferate when they are seeded in rat scaffold (Figure 5, J-N for C1 cells, and Supplemental Figure 11, J-M for C2 cells).

The formation of type I cells in vivo from type II cells is accompanied by a loss of the NKX 2.1 protein (1). Consistent with this pattern, some engrafted AETII cells acquired a flattened morphology and expressed the type 1 pneumocyte marker T $1 \alpha$ but lacked expression of NKX2.1 protein by costaining (Figure 5O). Moreover the number of T1 $\alpha$-positive cells significantly increased from $9.7 \%$ before cell seeding to $31.2 \%$ after culturing cells in the rat lung bioreactor. Conversely, the number of SPC-positive cells decreased from $98 \%$ before cell seeding to $72.3 \%$ after 7 days cultured in rat lung scaffold in bioreactor. Since type I cells are terminally differentiated cells and are not able to proliferate like type II cells, these observations may indicate that the ECM cues support a epithelial populations and that iPSC-AETII are able to differentiate to type I cells within the lung scaffold (Figure 5P). All of the iPSC-AETII cells were negative for CCSP and p63 by FACS analysis after 7 days of culture in the rat lung scaffold (Figure 5P).

In parallel experiments, iPSC-derived AETII were cultured on sections of rat and human lung matrix. To prepare sections of the acellular lung matrix, we treated lungs from adult donors using a procedure similar to that previously described $(8,33)$. Then, iPSCAETII cells were seeded onto $600-\mu \mathrm{m}$-thick sections of acellular human lung matrices and cultured in 6-well plates in SAGM with $1 \%$ FBS for 7 days. The iPSC-AETII cells adhered well to matrix surfaces and were initially distributed widely throughout alveoli in the matrix in both the rat and human decellularized lung sections. Many of the cells still showed AETII morphology, and there was robust expression of SPC and NKX2.1 (Figure 6, A-F, on human lung sections, and Figure $6, \mathrm{H}-\mathrm{K}$ on rat lung sections), while some engrafted AETII cells acquired a flattened morphology and expressed the AETI marker, $\backslash \mathrm{T} 1 \alpha$ (Figure $6 \mathrm{C}$ on human lung sections and Figure 6J on rat lung section). iPSC-derived AETII were able to proliferate on both rat and human lung sections, as determined by immunostaining for PCNA and caspase-3 (Figure 6G on human lung sections, and Figure 6, $\mathrm{L}-\mathrm{O}$ on rat lung section).

As a control for these experiments, isolated human type II cells from adult human lung (hAETII cells) were also seeded onto decellularized human lung sections. As with the iPSC-AETII cells, many of the cells with hAETII morphology expressed SPC and NKX2.1, and some of the hAETII cells gave rise to T $1 \alpha$-positive cells (Figure $6, \mathrm{P}-\mathrm{U})$. Moreover, they were able to replicate on the human scaffold sections, and the majority of cells expressed PCNA, while they displayed few markers of apoptotic cell death, as determined by immunostaining for caspase-3 (Figure 6V).

\section{Discussion}

Lung epithelia remain among the least-studied lineages to be derived from ESCs and iPSCs in vitro to date, and few research groups have reported on the differentiation toward lung epithelium $(5,14-16)$. Conditions for directing hESCs or iPSCs to differentiate along an alveolar epithelial lineage with homogeneity are not yet fully defined, and most protocols generate a mixed population of alveolar epithelium from hESCs or iPSCs. In the mouse, a NKX2.1:GFP reporter was used to isolate cells committed to the lung fate, which were then amenable to further differentiation (1). Moreover, in heterogeneous cultures of differentiating ESCs, induction of late markers of development such as surfactant protein C (SPC) have been reported, but their expression appears to be stochastic, and the cells expressing these markers have been difficult to expand $(1,14,15,17$, $18,20)$. In the present study, we have shown an efficient and consistent, step-wise differentiation method to generate DE, $\mathrm{AFE}$, and subsequently, a relatively homogeneous population of hAETII cells from 2 different iPSC clones (C1, reprogrammed from fetal lung fibroblasts; and $\mathrm{C} 2$, reprogrammed from neonatal fibroblasts). Interestingly, both iPSC clones yield similar results and had similar efficiency in differentiating toward DE, AFE, AETII and AETI cells, suggesting this protocol can be generalized to other iPSC lines from other sources. Unlike isolated human type II cells, however, these iPSC-AETII cells are capable of proliferating for several passages without losing AETII cellassociated markers, such as SPC, SPA, and mucin-1, and can be used to generate tens of millions of cells with which to seed the acellular matrix scaffold. The ability to "scale up" a progenitor population will be particularly valuable when translating these technologies for use in producing human tissues and allows for the possibility of using autologous iPSC-derived cells in future lung bioengineering work.

Under appropriate culture conditions, iPSC-derived AETII cells seeded into either rat decellularized lung bioreactors or onto decellularized rat or human lung sections behave similarly to isolated human type II cells. In these studies, the iPSC-AETII cells were able to diffusely repopulate alveolar lung structures. Although the majority of seeded iPSC-AETII cells still showed approximate AETII morphology, the percentages of T1 $\alpha$-positive cells increased to approximately $30 \%$ within the rat lung matrix over a 7-day culture period. It is possible that this shift in expression is a differentiating effect of correct cell-matrix interactions. Although type II cells are differentiated cells, these cells nonetheless retain a level of plasticity. Following peripheral lung injury, type II cells undergo proliferation and differentiation toward the type I phenotype. In fact, type II cells are considered to be putative alveolar stem cells and are crucial to the natural regenerative process of the alveoli $(20,29)$. The interaction between AETII cells and the lung matrix may have had an especially great effect, since it is likely that the AETII cells are still in a progenitor state able to give rise to T1 $\alpha$ cells when they adhere in regions where native lung contains type I cells and maintain a type II cell phenotype in regions where type II cells are typically be found. Since type I cell markers were not detected in the majority of the cells cultured within lung scaffold in bioreactor, it is possible that additional stimuli, such as cyclic stretch or exposure to an air-liquid interface, may be necessary to promote expression of more type I alveolar markers $(25,37,38)$.

Future work will concentrate on the production of more types of lung progenitors from iPSCs in order to expand the types of cells that can be used for lung engineering purposes. In addition, longer bioreactor culture time points (e.g., 14 or 21 days) would likely allow the epithelial population to further expand within the matrix and adopt more characteristic (and functional) phenotypes. Production of functional epithelium would also be aided by exposure to an air-liquid interface or by experiencing the stretch produced by breathing with either air or liquid (16). Although our results represent only an initial step toward the ultimate goal of generating fully functional lungs in vitro, these studies suggest that in vitro lung regeneration from autologous cells may be a viable strategy for tissue repair and cell therapy applications. 
iPSC-derived AETII on human lung slices

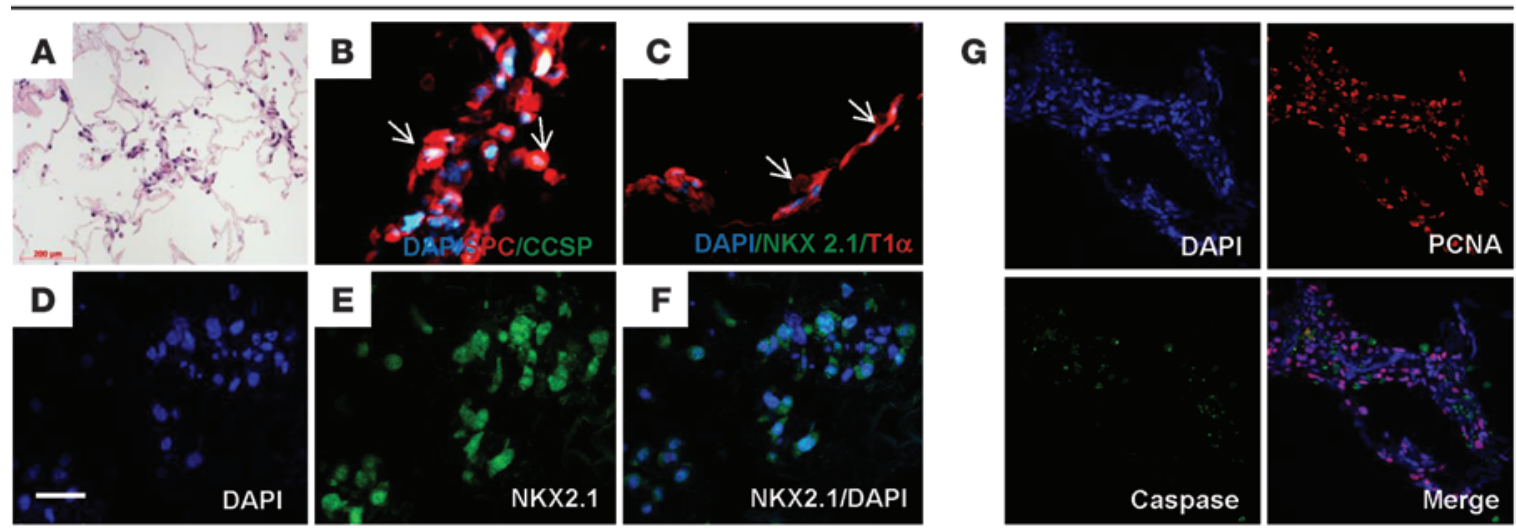

iPSC-derived AETII on rat lung slices

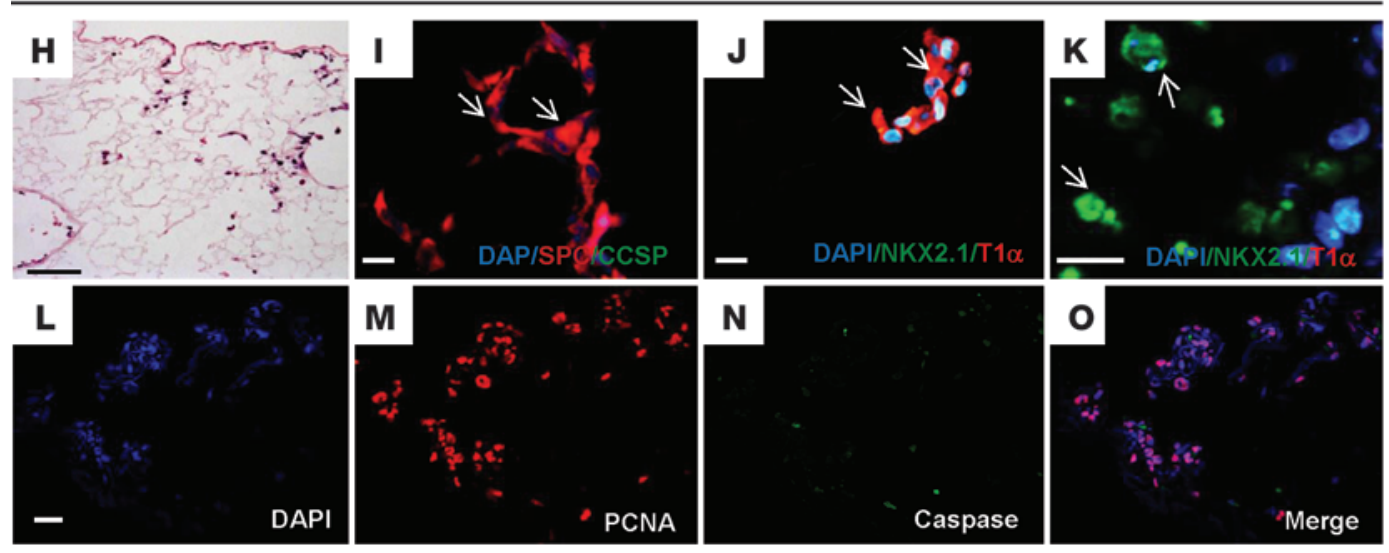

Human AETII on human lung slices

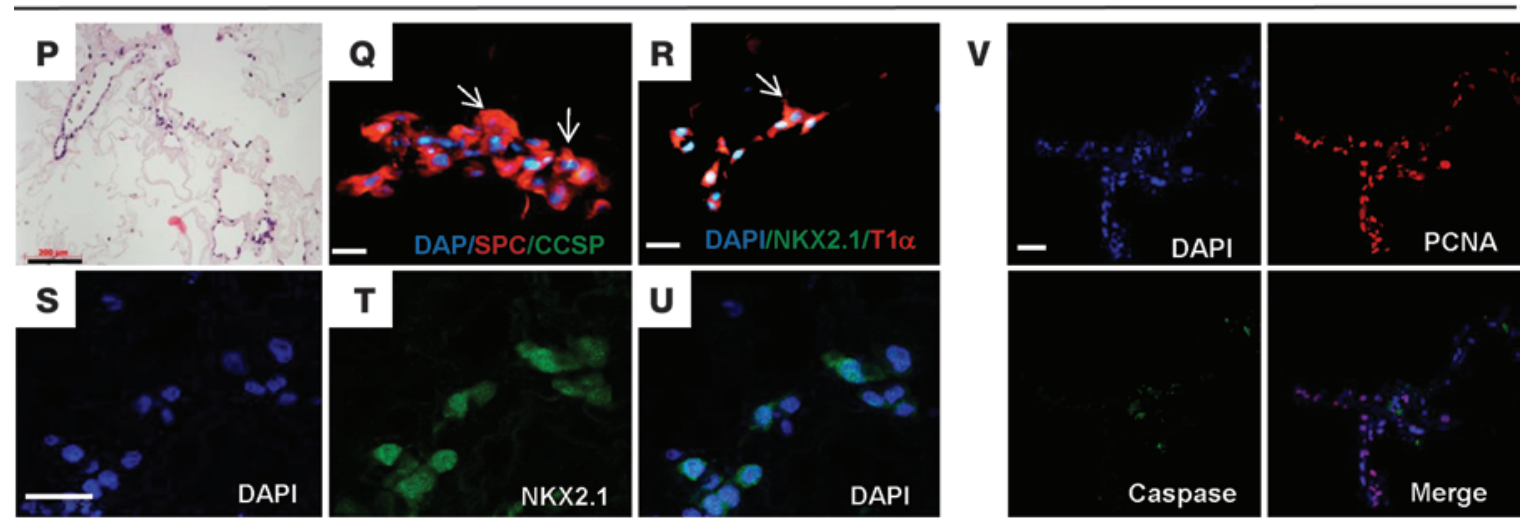

\section{Figure 6}

iPSC-derived AETII (C1 clone) adhere to sections of acellular rat and human lung matrix. (A-G) iPSC-AETII on human lung sections at day 7. (A) H\&E. Scale bar: $200 \mu \mathrm{m}$ (B) Immunostaining for SPC and CCSP. (C) Immunostaining for NKX2.1 and T1 $\alpha$ (arrows indicates cells positive for SPC in B and T1 $\alpha$ in C. Scale bar: $63 \mu \mathrm{m}$. (D-F) Immunostaining for NKX2.1. (D), DAPI, (E) NKX2.1, (F) merging. Scale bar: $50 \mu \mathrm{m}$. (G) Caspase and PCNA immunostaining. Scale bar: $49 \mu \mathrm{m}(\mathbf{H}-\mathbf{O})$ iPSC-derived AETII cultured on rat lung sections for 7 days. (H) H\&E. Scale bar: $200 \mu \mathrm{m}$. (I) Immunostaining for SPC and CCSP. (J, K) Immunostaining for NKX2.1 and T1 $\alpha$ (arrows indicate cells positive for SPC in I, T1 $\alpha$ in J, and NKX2.1 in K). Scale bars: $63 \mu \mathrm{m}$. (L) DAPI staining, (M) immunostaining for PCNA, and (N) caspase, (O) merge. Scale bar: $50 \mu \mathrm{m}$. (P-V) Native human AETII cells, isolated from fresh adult human lung, cultured on human lung sections for 7 days. (P) H\&E. Scale bar: $200 \mu \mathrm{m}$. (Q) Immunostaining for SPC and CCSP. Scale bar: $63 \mu \mathrm{m}$ (R) Immunostaining for NKX2.1 and T1 $\alpha$. Scale bar: $63 \mu \mathrm{m}$ (arrows indicate cells positive for SPC in $\mathbf{Q}$ and T1 $\alpha$ in $\mathbf{R}$ ). (S-U) Immunostaining for NKX2.1. (S) DAPI, (T) NKX2.1, (U) merge. Scale bar: $50 \mu \mathrm{m}$. (V) Caspase and PCNA immunostaining. Scale bar: $49 \mu \mathrm{m}$. 


\section{Methods}

Chemical and reagents. Mouse embryonic fibroblasts (MEFs) (GSC-6201) were purchased from GlobalStem, Matrigel (354277) was purchased from BD, recombinant human WNT3a $(5036 \mathrm{WN})$ was purchased from R\&D. Dispase (07923) was obtained from Stem Cell Technology. Keratinocyte GF (KGF) (PHG0094), FGF-10 (PHG0204), EGF (PHG0311), human basic FGF (bFGF) (13256-029), NOGGIN (PHC1506), activin A (PHG9014), knockout serum replacement (108280280), and Superscript First Strand Synthesis System for RT-PCR (18080-051) were purchased from Invitrogen. DMEM: Nutrient Mixture F-12 (DMEM/F-12) (11330032), DMEM (11905-092), RPMI 1640 (11875-093), IMDM (12440-053), nonessential amino acids (1140-050), L-glutamine (25030164), sodium pyruvate (11360-070), 2-mercaptoethanol (21985-023), B27 supplement (17504-044), Trypsin (25200-056), penicillin-streptomycin (15140122), and FBS were purchased from Life Technology (Gibco). Retinoic acid (R2625), gelatin (G1393), SB431542 (S4317), human ECM protein (E0282), IWR-1 (I0161), human collagen type I (C7624), and IV (C7521), human fibronectin (F0895), human ECM protein (E0282), CHAPs (C3023), benzonase (E1014), sodium deoxycholate (D6750), elastase (E8140), and Triton X-100 (T9284), were purchased from Sigma-Aldrich. Sodium nitroprusside dihydrate (71778) was obtained from Fluka. DNase I (LS006333) was purchased from Worthington Biochemical Corp. Small Airway Growth Medium (SAGM) (CC-3119) and amphotericin B (17836R) were purchased from Lonza. The human SPC ELISA kit (E01S0168) was obtained from Life Science Advanced Technologies Inc. Peracetic acid (PAA) (P05020) was obtained from Pfalz \& Bauer. FBS (SH30071.03) was obtained from Hyclone. iQ SYBR Green Supermix (170-8882) was obtained from Bio-Rad. All antibodies were used in this study are listed in Supplemental Table 1.

Cultivation of human iPSCs. The human iPSC lines, iPSC (IMR90, C1) and iPSC (neonatal foreskin, C2), utilized here were provided by James A. Thomson (Department of Anatomy, University of Wisconsin-Madison, Madison, Wisconsin, USA) (11). Both human iPSC lines were generated by lentiviral transduction of isolated human skin fibroblasts (IMR90, C1 clone) and neonatal foreskin fibroblast (C2 clone) with OCT-4, SOX2, Nanog, and lin 28 genes. These induced pluripotent human stem cells have been extensively characterized; they have normal karyotypes and telomerase activity, express cell-surface markers and genes that characterize human ESCs, and maintain the developmental potential to differentiate into advanced derivatives of all 3 primary germ layers (11). Both lines were cultured and maintained as described previously (11). Briefly, iPSCs were propagated on irradiated MEF feeder layers in DMEM-F12 medium supplemented with 20\% knockout serum replacement, $4 \mathrm{ng} / \mathrm{ml} \mathrm{bFGF}, 1 \mathrm{mM}$ glutamine, $1 \% \mathrm{mM}$ nonessential amino acids, and $0.1 \mathrm{mM} \beta$-mercaptoethanol at $37^{\circ} \mathrm{C}, 5 \% \mathrm{CO}_{2}$, and $90 \%$ $95 \%$ humidity, with medium changes every day. Undifferentiated iPSCs were passaged every $4-5$ days onto fresh feeders by mechanical dissociation using a Stem Cell Cutting Tool (VWR).

In vitro differentiation of iPSCs into AETII cells. iPSCs were differentiated into alveolar epithelium in a directed differentiation protocol via $\mathrm{DE}$ and AFE. iPSCs were differentiated into DE under conditions described previously $(39,40)$. Briefly, hiPSCs were cultured in RPMI 1640 medium supplemented with $100 \mathrm{ng} / \mathrm{ml}$ activin A, $2 \mathrm{mM} \mathrm{L-glutamine,} \mathrm{and} \mathrm{1 \%} \mathrm{antibiotic-}$ antimycotic for 48 hours; $1 \times B 27$ supplement, $0.5 \mathrm{mM}$ sodium butyrate, and $0.1 \%$ FBS were added into the same medium, and the cells were cultured for another 4 days, with daily medium changes (41).

DE generated by exposure to activin A were trypsinized, reseeded at a ratio of 1:1-2 on human ECM protein-coated plates, and differentiated into AFE with IMDM + 5\% FBS, $2 \mathrm{mM}$ L-glutamine, $1 \mathrm{mM}$ nonessential amino acids, $1 \%$ antibiotic-antimycotic supplemented with $200 \mathrm{ng} / \mathrm{ml}$ NOGGIN, and $10 \mathrm{mM} \mathrm{SB}-431542$ for 2 days $(1,14)$.
AFE cells were maintained in IMDM differentiation medium with $10 \%$ FBS, $2 \mathrm{mM}$ L-glutamine, $1 \mathrm{mM}$ nonessential amino acids, $1 \%$ antibioticantimycotic, retinoic acid $(0.5 \mu \mathrm{M}), \mathrm{FGF}-10(10 \mathrm{ng} / \mathrm{ml}), \mathrm{EGF}(10 \mathrm{ng} / \mathrm{ml})$, Wnt3a (100 ng/ml), and KGF (10 ng/ml each) for 10-14 days. Cells were maintained in SAGM culture medium (Lonza) plus 1\% FBS until seeding into lung matrices $(1,14)$.

Differentiated cells at day 22 were then maintained in DMEM medium with $10 \% \mathrm{FBS}, 2 \mathrm{mM}$ L-glutamine, $1 \mathrm{mM}$ nonessential amino acids, $1 \%$ antibiotic-antimycotic, and $100 \mathrm{mM}$ IWR-1 for 7 days.

Isolation of human type II cells. AETII cells were isolated from human lungs rejected for transplant as previously described (31). Briefly, the right middle lobe was cannulated through the main stem bronchus and removed from the rest of the lung. The distal airspaces were lavaged 6-10 times using a $\mathrm{Ca}^{2+}$ - and $\mathrm{Mg}^{2+}$-free solution $(0.5 \mathrm{~mm}$ EGTA, $140 \mathrm{~mm} \mathrm{NaCl}, 5 \mathrm{~mm}$ $\mathrm{KCl}, 2.5 \mathrm{~mm} \mathrm{Na}_{2} \mathrm{HPO}_{4}, 10 \mathrm{~mm}$ HEPES, and $6 \mathrm{~mm}$ glucose) and lavaged 3 times with a modified version of this solution (no glucose, $2.0 \mathrm{~mm} \mathrm{CaCl}$, and $1.3 \mathrm{~mm} \mathrm{MgSO}_{4}$ ). Elastase (13 units $/ \mathrm{ml}$ ) was instilled into the distal airspaces and incubated at $37^{\circ} \mathrm{C}$ for 30 minutes. Isolated cells were resuspended in DMEM and decanted onto PBS- and DMEM-rinsed Petri dishes coated with human IgG antibody. After 60 minutes at $37^{\circ} \mathrm{C}$, nonadherent AETII cells were incubated with a monoclonal antibody against fibroblasts (AS02) and pan-mouse IgG Dynabeads for removal by magnet. AETII cells were resuspended in DMEM containing 10\% FBS, amphotericin B, ceftazidime, tobramycin, and vancomycin.

Flow cytometry and immunochemistry. DE, AFE, and iPSC-AETII cell populations were assessed by immunofluorescence or/and flow cytometry before differentiation during the induction of DE and AFE and iPSCAETII and after cultivation in the decellularized lung matrix.

For immunostaining, cells were washed with PBS, fixed in $4 \%$ paraformaldehyde for 20 minutes at room temperature (RT) and permeabilized with $0.1 \%$ Triton X-100 in PBS for 15 minutes at RT. Cells were blocked in $3 \%$ BSA in PBS for 60 minutes at RT and incubated with primary antibody overnight at $4{ }^{\circ} \mathrm{C}$. The next day, cells were washed with PBS and incubated with secondary antibody for 2 hours at RT. After washing, the cells were incubated with DAPI (1:1000) nuclear stain.

Paraffin sections of cell-seeded lung scaffolds were stained with H\&E. Additional sections were permeabilized with $0.2 \%$ Triton X-100 for 15 minutes after heat-mediated citric acid antigen retrieval and blocked with $5 \%$ BSA for 1 hour at RT. Primary antibodies were applied overnight at $4{ }^{\circ} \mathrm{C}$. Sections were incubated with secondary antibodies for 1 hour at RT, rinsed, treated with DAPI for 1 minute, and mounted with PVA-DABCO cover slipping solution. Stained cells and slides were imaged with a Zeiss Axiovert 200M inverted microscope and a Hamamatsu camera.

For flow cytometry, cells were dissociated into single-cell suspensions by incubation with $0.25 \%$ trypsin for 2 minutes and fixed (Fixation/Permeabilization Kit, BD Biosciences). After blocking for 30 minutes on ice, the cells were incubated with primary antibody in blocking solution for 30 minutes on ice. The cells were resuspended in $350 \mu \mathrm{l}$ of Perm/Wash buffer after incubation with conjugated secondaries for 30 minutes on ice, washed twice, and analyzed by flow cytometry. See Supplemental Table 1 for antibody information.

Real time $q R T-P C R$. Total RNA was extracted using the RNeasy Mini Kit from QIAGEN, following the manufacturer's instructions. First-strand cDNA was synthesized with random hexamers as primers, using SuperScript First-Strand Synthesis System according to the manufacturer's protocol (Invitrogen). Each sample was run in triplicate with iQ SYBR Green Supermix (Bio-Rad). PCR conditions included an initial denaturation step of 4 minutes at $95^{\circ} \mathrm{C}$, followed by 40 cycles of PCR consisting of 15 seconds at $95^{\circ} \mathrm{C}, 30$ seconds at $60^{\circ} \mathrm{C}$, and 30 seconds at $72^{\circ} \mathrm{C}$. Average Ct values from the triplicate PCR reactions for a gene of interest (GOI) 
were normalized against average GAPDH Ct values from the same cDNA sample. Fold change of GOI transcript levels between sample A and sam-

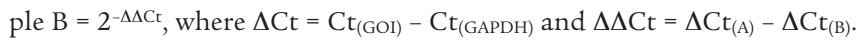
See Supplemental Table 2 for primers.

Transmission electron micrographs. Cell samples were prepared following a modified protocol from Schmiedl et al. (42). Briefly, native human AETII and iPSC-AETII cells were fixed at $37^{\circ} \mathrm{C}$ with a $2.5 \%$ glutaraldehyde $/ 2.0 \%$ paraformaldehyde mixture in $0.2 \mathrm{M}$ sodium cacodylate for 30 minutes, followed by 2 hour incubation at $4{ }^{\circ} \mathrm{C}$. The samples were dehydrated following a standard ethanol series. The samples were postprocessed by $\mathrm{OsO}_{4}$ fixation and en block uranyl acetate staining. Then 70 - to 80 -nm sections were taken and incubated in uranyl acetate and lead citrate for increased contrast. Images were taken using a Philips Tecnai transmission electron microscope.

Preparation of decellularized ECM scaffolds. Three-month-old Fischer or Sprague-Dawley rats were anesthetized with sodium pentobarbital according to the guidelines set forth by the American Veterinary Medical Association (60 mg/kg i.p.). Lung ECM scaffolds were prepared as previously described $(8,43)$. Lungs were perfused with heparin $(50 \mathrm{U} / \mathrm{ml}$, Sigma-Aldrich) in PBS and removed with the heart and trachea. The pulmonary artery and trachea were cannulated, and the lungs were perfused through the pulmonary artery with sodium nitroprusside ( $1 \mathrm{ml} / \mathrm{ml}$; Fluka) before being treated with decellularization solution (8 mM CHAPS, $1 \mathrm{M}$ $\mathrm{NaCl}, 5 \mathrm{mM}$ EDTA in PBS) for $2-3$ hours at $37^{\circ} \mathrm{C}$. Scaffolds were treated with benzonase endonuclease ( $90 \mathrm{U} / \mathrm{ml}$; Sigma-Aldrich) for 1 hour at $37^{\circ} \mathrm{C}$, followed by extensive rinsing with PBS, antibiotics, and antimycotics.

Human lungs were obtained from beating-heart donors or warm autopsy as arranged through Gift of Life Michigan, and were decellularized as we have recently described (33). Lung samples were agitated in sterile deionized, distilled water and incubated in $0.1 \%$ Triton X-100 for cell lysis. Samples were washed with sterile PBS, incubated with $2 \%$ sodium deoxycholate, and washed again. Lungs were incubated in $1 \mathrm{M} \mathrm{NaCl}$ to lyse residual nuclei. After decanting $\mathrm{NaCl}$, tissues were rinsed and incubated with $30 \mu \mathrm{g} / \mathrm{ml}$ DNAse in $1.3 \mathrm{mM} \mathrm{MgSO}_{4}$ and $2 \mathrm{mM} \mathrm{CaCl}_{2}$. The DNAse solution was decanted, and tissues were washed with sterile PBS (33).

Culture of cells on rat lung ECM scaffolds. Rat scaffolds were mounted in the bioreactor as described previously (8). Cannulas were connected to tubing loops to provide perfusion and introduction of cells to the scaffold. Forty million iPSC-AETII cells were suspended in 3-5 $\mathrm{ml}$ of culture medium (SAGM-1\% FBS) and introduced into the airway compartment; perfusion was initiated at $1 \mathrm{ml} / \mathrm{min}$ immediately after cell seeding. In additional experiments, $6 \times 10^{6}$ native human AETII cells - isolated from human lung - were introduced into the upper right lobe of a decellularized rat lung. The full volume of culture medium was changed once at day 3 or 4 , and samples were harvested at days 1, 3, and 7 and saved for histology. In parallel experiments, iPSC-AETII cells were seeded onto sections of decellularized rat lung at a concentration of $1.5 \times 10^{5}$ cells/slice in SAGM-1\% FBS medium for 7 days. Finally, $3 \times 10^{5}$ of either AETII cells or native human AETII cells were transferred onto decellularized human lungs slices in SAGM-1\% FBS and cultured for 1 week; medium was changed every other day.

ELISA for SPC. ELISA was performed on cell culture medium collected during iPSC-AETII differentiation to quantify secreted SPC (Life Science Advanced Technology) according to the manufacturer's instructions. SPC values were normalized to the total number of cells.

Western blotting. Cells were incubated in RIPA buffer supplemented with protease inhibitors (Complete Mini; Roche,) on ice for 30 minutes. Protein concentration was determined from cell lysates using a bicinchoninic acid protein assay (Thermo Fisher Scientific). Cell lysates were denatured, and equal amounts of protein per sample were subjected to SDS-PAGE and immunoblotting as described previously (8). HRP-conjugated goat anti-mouse and goat anti-rabbit secondaries were detected by enhanced chemiluminescence.

Proliferation assay. To assess cell proliferation within the lung scaffold, lungs seeded and cultured with AETII for 3 days and 7 days were fixed in $4 \%$ PBS-buffered paraformaldehyde ( $\mathrm{pH} 7.4$ ) and postfixed with $70 \%$ EtOH. The immunocytochemical staining against human caspase and PCNA was performed as described in the immunostaining section. The images were visualized with a Zeiss Axiovert 200M inverted microscope and imaged with a Hamamatsu camera. The percentage of positive nuclear staining was calculated based on total cell numbers in 3 high-power fields.

Statistics. Statistics were done with Origin (OriginLab). The data were expressed as mean \pm SEM (all error bars represent \pm SEM). Unpaired, 2 -tailed Student's $t$ tests were performed to evaluate whether the 2 groups were significantly different from each other. $P$ values of less than 0.05 (2-tailed) were considered statistically significant.

Study approval. All animal experiments were performed with the approval of the IACUC of Yale University. All studies using human lung tissue were approved by the University of Michigan and the University of North Carolina institutional review boards.

\section{Acknowledgments}

We gratefully acknowledge James Thomson for providing human iPSC clones. This work was supported by United Therapeutics Inc. United Therapeutics did not affect the content or conclusions contained in this manuscript. Work was also supported by NIH U01 HL111016 and R01 HL098220 (both to L.E. Niklason). J.J. Mendez is supported by T32 GM086287.

Received for publication January 14, 2013, and accepted in revised form August 15, 2013.

Address correspondence to: Laura E. Niklason, Departments of Anesthesia and Biomedical Engineering, Yale University, New Haven, Connecticut 06520, USA. Phone: 203.737.1422; Fax: 203.737.1484; E-mail: laura.niklason@yale.edu.
1. Longmire TA, et al. Efficient derivation of purified lung and thyroid progenitors from embryonic stem cells. Cell Stem Cell. 2012;10(4):398-411.

2. Petersen TH, Calle EA, Niklason LE. Strategies for lung regeneration. Mater Today. 2011;14(5):196-201.

3. Nichols JE, Niles JA, Cortiella J. Production utilization of acellular lung scaffolds in tissue engineering. J Cell Biochem. 2012;113(7):2185-2192.

4. McCurry KR, et al. Lung transplantation in the United States, 1998-2007. Am J Transplant. 2009; 9(part 2):942-958.

5. Wang D, Haviland DL, Burns AR, Zsigmond E, Wetsel RA. A pure population of lung alveolar epithelial type II cells derived from human embryonic stem cells. Proc Natl Acad Sci US A. 2007;104(11):4449-4454.

6 . Tesei A, et al. Isolation of stem/progenitor cells from normal lung tissue of adult humans. Cell Prolif. 2009; 42(3):298-308.

7. Fujino $\mathrm{N}$, et al. A novel method for isolating individual cellular components from the adult human distal lung. Am J Respir Cell Mol Biol. 2012; 46(4):422-430.

8. Petersen TH, et al. Tissue-engineered lungs for in vivo implantation. Science. 2010;329(5991):538-541.

9. Badylak SF, Weiss DJ, Caplan A, Macchiarini P. Engineered whole organs and complex tissues. Lancet. 2012;379(9819):943-952.

10. Kotton DN. Next-generation regeneration: the hope and hype of lung stem cell research. Am J Respir Crit Care Med. 2012;185(12):1255-1260.

11. Takahashi $\mathrm{K}$, et al. Induction of pluripotent stem cells from adult human fibroblasts by defined fac- tors. Cell. 2007;131(5):861-872.

12. $\mathrm{Yu} \mathrm{J}$, et al. Induced pluripotent stem cell lines derived from human somatic cells. Science. 2007;318(5858):1917-1920.

13. Nishikawa S, Goldstein RA, Nierras CR. The promise of human inducedpluripotent stem cells for research and therapy. Nat Rev Mol Cell Biol. 2008;9(9):725-729.

14. Green MD, et al. Generation of anterior foregut endoderm from human embryonic and induced pluripotent stem cells. Nat Biotechnol. 2011;29(3):267-272.

15. Mou H, et al. Generation of multipotent lung and airway progenitors from mouse ESCs and patient-specific cystic fibrosis iPSCs. Cell Stem Cell. 2012;10(4):385-397.

16. Van Haute L, De Block G, Liebaers I, Sermon K, 
De Rycke M. Generation of lung epithelial-like tissue from human embryonic stem cells. Respir Res. 2009; 10:105.

17. Ali NN, et al. Derivation of type II alveolar epithelial cells from murine embryonic stem cells. Tissue Eng. 2002;8(4):541-550.

18. Rippon HJ, Polak JM, Qin M, Bishop AE. Derivation of distal lung epithelial progenitors from murine embryonic stem cells using a novel threestep differentiation protocol. Stem Cells. 2006; 24(5):1389-1398.

19. Samadikuchaksaraei A, et al. Derivation of distal airway epithelium from human embryonic stem cells. Tissue Eng. 2006;12(4):867-875.

20. Banerjee ER, Laflamme MA, Papayannopoulou T, Kahn M, Murry CE, Henderson WR Jr. Human embryonic stem cells differentiated to lung lineagespecific cells ameliorate pulmonary fibrosis in a xenograft transplant mouse model. PLoS One. 2012; 7(3):e33165.

21. Kadzik RS, Morrisey EE. Directing lung endoderm differentiation in pluripotent stem cells. Cell Stem Cell. 2012;10(4):355-361.

22. Yasunaga $M$, et al. Induction and monitoring of definitive and visceral endoderm differentiation of mouse ES cells. Nat Biotechnol. 2005;23(12):1542-1550.

23. Reilly GC, Engler AJ. Intrinsic extracellular matrix properties regulate stem cell differentiation. J Biomech. 2010;43(1):55-62.

24. Lin YM, Zhang A, Rippon HJ, Bismarck A, Bishop AE. Tissue engineering of lung: the effect of extracellular matrix on the differentiation of embryonic stem cells to pneumocytes. Tissue Eng Part A. 2010;
16(5):1515-1526.

25. Gutierrez JA, Gonzalez RF, Dobbs LG. Mechanical distension modulates pulmonary alveolar epithelial phenotypic expression in vitro. Am J Physiol. 1998;274(2 pt 1):L196-L202.

26. Chapman HA, et al. Integrin $\alpha 6 \beta 4$ identifies an adult distal lung epithelial population with regenerative potential in mice. J Clin Invest. 2011; 121(7):2855-2862.

27. Whitsett JA, Kalinichenko VV. Integrin $\alpha 6 \beta 4$ defines a novel lung epithelial progenitor cell: a step forward for cell-based therapies for pulmonary disease. J Clin Invest. 2011;121(7):2543-2545.

28. Soh BS, et al. CD166(pos) subpopulation from differentiated human ES iPS cells support repair of acute lung injury. Mol Ther. 2012;20(12):2335-2346.

29. Asselin-Labat ML, Filby CE. Adult lung stem cells and their contribution to lung tumourigenesis. Open Biol. 2012;2(8):120094.

30. Fehrenbach H. Alveolar epithelial type II cell: defender of the alveolus revisited. Respir Res. 2001; 2(1):33-46.

31. Bove PF, et al. Human alveolar type II cells secrete and absorb liquid in response to local nucleotide signaling. J Biol Chem. 2010;285(45):34939-34949.

32. Fujino $\mathrm{N}$, et al. Isolation of alveolar epithelial type II progenitor cells from adult human lungs. Lab Invest. 2011;91(3):363-378.

33. Booth AJ. Acellular normal and fibrotic human lung matrices as a culture system for in vitro investigation. Am J Resp Crit Care Med. 2012;186(9):866-876.

34. Daly $A B$, et al. Initial binding and recellularization of decellularized mouse lung scaffolds with bone marrow-derived mesenchymal stromal cells. Tissue Eng Part A. 2012;18(1-2):1-16.

35. Ott HC, et al. Regeneration and orthotopic transplantation of a bioartificial lung. Nat Med. 2010; 16(8):927-933.

36. Petersen TH, Calle EA, Colehour MB, Niklason LE. Bioreactor for the long-term culture of lung tissue. Cell Transplant. 2011;20(7):1117-1126.

37. Ostrowski LE, Nettesheim P. Inhibition of ciliated cell differentiation by fluid submersion. Expt Lung Res. 1995;21(6):957-970.

38. Alcorn D, Adamson TM, Lambert TF, Maloney JE, Ritchie BC, Robinson PM. Morphological effects of chronic tracheal ligation and drainage in the feta llamb lung. J Anat. 1977;123(pt 3):649-660.

39. Duan Y, et al. Differentiation and characterization of metabolically functioning hepatocytes from human embryonic stem cells. Stem Cells. 2010;28(4):674-686.

40. Kubo A, et al. Development of definitive endoderm from embryonic stem cells in culture. Development. 2004;131(7):1651-1662.

41. D’Amour KA, Agulnick AD, Eliazer S, Kelly OG, Kroon E, Baetge EE. Efficient differentiation of human embryonic stem cells to definitive endoderm. Nat Biotechnol. 2005;23(12):1534-1541.

42. Schmiedl A, Ochs M, Muhlfeld C, Johnen G, Brasch F. Distribution of surfactant proteins in type II pneumocytes of newborn, 14-day-old, adult rats: an immunoelectron microscopicand stereological study. Histochem Cell Biol. 2005;124(6):465-476.

43. Calle EA, Petersen TH, Niklason LE. Procedure for lung engineering. J Vis Exp. 2011;(49):pii:2651. 